\title{
Adverse selection, commitment and exhaustible resource
}

\author{
taxation
}

\begin{abstract}
This paper studies the contractual relationship between a government and a firm in charge of the extraction of an exhaustible resource. Governments design taxation scheme to capture resource rent and they usually propose contracts with limited duration and possess less information on resources than the extractive firms do. This article investigates how information asymmetry on costs and an inability to commit to long-term contracts affect tax revenue and the extraction path. This study gives several unconventional results. First, when information asymmetry exists, the inability to commit does not necessarily lower tax revenues. Second, under asymmetric information without commitment, an efficient firm may produce during the first period more or less than under symmetric information. Hence, the inability to commit has an ambiguous effect on the exhaustion date. Third, the modified Hotelling's rule is such that an increase in the discount factor does not necessarily reduce the first-period extraction.
\end{abstract}

Keywords: resource taxation; asymmetric information; commitment

\section{Introduction}

Natural resource revenues are substantial and developing economies rely heavily on the tax receipts they generate. According to the International Monetary Fund, Algeria's oil and gas revenues provides more than $95 \%$ of the country's export earnings and $60 \%$ of its budget. Today, diamonds account for $76 \%$ of Botswana's export revenue, $45 \%$ of the government revenue 
and $33 \%$ of the gross domestic product. Despite the resource curse $^{1}$, natural resources are a remarkable opportunity to foster development and reduce poverty. Nevertheless, their taxation comes with significant challenges for many low-income countries. In regions that have yet to develop their natural resources, it will not be possible to establish national mining companies and exclude international companies and their expertise. Many governments delegate the exploitation of their natural resources to international private firms. However, designing the fiscal instrument is not an easy task since governments may lack information. As Collier and Venables (2010) and Humphreys et al. (2007) point out, governments usually possess less information on resource's geological settings and commercial value than extractive companies. Consequently, according to Boadway and Keen (2009), firms may understate resource stocks or overstate their costs. Similarly, Osmundsen (2009) claims that companies may act strategically by employing datasets and measurement methods that best serve their interests. As a result, governments should design taxation schemes that take extractive firms' strategic behavior into account.

Different legal regimes such as concessionary systems or contractual systems (production sharing agreement and service contracts) can be used to delegate the development, the exploration and the extraction of exhaustible resources to private firms. Although these legal regimes are conceptually different from each other, they have the common feature of being signed for a limited duration. Governments do not commit to a taxation scheme that will be implemented until the exhaustion of the resource. Resources are developed through a succession of contracts. In fact, in modern concessions the contract duration has been shorten. For instance, in the Turkish Petroleum Code, Article 65, specifies that a license is to be granted for twenty years with a possibility of two extensions for no more than ten years each if certain conditions have been met. ${ }^{2}$ The inability to commit to long-term contracts has major effects

\footnotetext{
${ }^{1}$ The resource curse states that countries with an abundance of natural resources tend to have less economic growth and worse development outcomes than countries with fewer natural resources (see Sachs and Warner (2001), Kolstad and Wiig (2009) or Robinson et al. (2006))

${ }^{2}$ Another example is the development of alumina in the Republic of Guinea. In October 2004, the Global Alumina Products Corporation and the Ministry of Mines and Geology signed a contract for the development of an alumina refinery in Conakry. This mining concession has an initial term of 25 years and may be renewed for 25 years. After these 50 years, a succession of ten year contracts is possible.
} 
on optimal taxation schemes (Farhi et al. (2012), Brito et al. (1991) or Dillen and Lundholm (1996)) especially with private information. Indeed, without commitment, if a regulated firm has private information, the regulator updates its belief at the end of each contract. If the parameter that is subject to information asymmetry is correlated over time, a firm knows that revealing its information jeopardizes the informational rents it receives in subsequent periods. This is the so-called ratchet effect. Therefore, a firm must receive a large upfront payment to reveal information. This payment increases with the discount factor: if a firm values the future highly, it must be significantly compensated to reveal its information. As pointed out by previous studies ${ }^{3}$, this has two consequences. First, an inefficient firm has more incentives to misrepresent its type when the discount factor is high. If so, both incentive constraints should bind. Second, the regulator is more willing to implement during the first period pooling or semi-separating contracts rather than full-separating contracts when the discount factor is high since separating firms becomes more costly.

In a nutshell, this paper studies the contractual relationship between a government and an extractive firm whose type, that is extraction efficiency, is private information. We only consider one firm, but two types of firms (efficient or inefficient) and we assume that the government can only delegate the extraction to this firm. This article investigates how asymmetric information on cost, an inability to commit to long-term contracts and a limited stock of resources affects the extraction path and the tax revenue. We pay particular attention to the discount factor and its effects on the type of contracts implemented (separating, semi-separating or pooling contracts) and on the extraction path. If the government is able to commit, it proposes in the first period the contracts for all subsequent periods and sticks to them. On the opposite, without commitment, the government proposes a contract for a given duration, and proposes at the end of this contract another one, and so on until the stock is exhausted or until the extraction is no more profitable. To simplify the analysis, we assume that each contract only lasts one period, in other words, each contract only regulates one extraction decision. We

\footnotetext{
${ }^{3}$ See for instance, Freixas et al. (1985), Laffont and Tirole (1988), Laffont and Tirole (1990) and Dionne and Fluet (2000).
} 
consider a government that maximizes the tax revenue without taking into account the firm's profit or the consumer surplus. Indeed, resource-rich countries delegate resource extraction to foreign companies and export a significant share of their resources. Finally, we assume that the regulator offers during the first period a menu of two contracts, or a pooling contract and we use the revelation principle (see Bester and Strausz (2001) for a discussion on these assumptions).

Introducing adverse selection into non-renewable resources models implies three types of dynamics. The first one is related to the exhaustion of the resource, since when the stock is exhausted future choices are constrained by current choices. The second one is related to the information, since the regulator updates its belief on the firm's type. The third one is related to the costs, since the size of the stock may affect the extraction costs. To our knowledge, only a few papers study how information asymmetry affects the taxation scheme, the extraction path and thus Hotelling's rule. ${ }^{4}$ This rule is an arbitrage condition for the use of resources over different periods of time and is central in non-renewable resources theories. It describes how the price of resource should evolve along an optimal extraction path and requires the rent (net price or marginal net benefits) grow over time at the rate of discount. This rule implies that an increase in the discount factor decreases current extraction and increases future extraction. The main contributions studying resource taxation with asymmetric information are summarized as follows. Gaudet et al. (1995) consider two periods and information asymmetry on the extraction costs. They show that when it is not optimal for some types of firms to exhaust the stock, the most efficient firm may produce more than the first best. Despite the noncommitment assumption, they neutralize the dynamics related to the information by assuming that the parameter that is subject to information asymmetry is not correlated over time. Hung et al. (2006) relax the two-period assumption, and find that information asymmetry on costs extends the contract duration. They also show that just before exhaustion, firms may produce a higher quantity than the first best. Despite a constant efficiency parameter, they also neutralize the effect of the information flow by assuming that the government commits to long-term

\footnotetext{
${ }^{4}$ Gaudet and Lassere (2015) propose an interesting literature review on the subject
} 
contracts. In contrast, Osmundsen (1998) assumes that as the stock decreases the extraction costs increases. He considers a two-period model where a firm has private information on the size of the stock and on the extraction costs. He finds that at each period, the most efficient firm should produce the same as under symmetric information (the first best). On the contrary, due to the trade-off between informational rent and productive efficiency, an inefficient firm should produce less than the first best. Osmundsen (1998) neutralizes the dynamics related to the exhaustion and the effect related to the information flow by assuming commitment and an asymptotic production cost function. More recently, Martimort et al. (2018) extend the paper by Osmundsen (1998) by considering an infinite time horizon.

This study defines the optimal taxation scheme taking into account two imperfections observed in reality: private information and a lack of commitment. We study in a two-period model two of the dynamic effects (resource and information) by assuming that there is no commitment and that the parameter that is subject to information asymmetry is constant. This paper's contribution is twofold. First, it extends the contract theory literature on noncommitment by introducing a stock constraint. We show that if the discount factor and the stock are both relatively low, only an efficient firm misrepresents its type, and both firms exhaust the stock during the second period, if so, the extraction path and tax revenue are the same with and without commitment. This contrasts with the traditional result that an inability to commit lowers tax revenue. Moreover, the stock constraint implies that the first period distortion affects the second-period production possibilities. Hence, during the second period, firms may produce more than the first best, the latter being too low because of the stock constraint. Second, the paper contributes to the literature on resource taxation with information asymmetry by focusing on informational dynamics. The main difference with the previous studies is that the regulator can use the information to design the future contracts. We show that without commitment, the efficient firm's first-period extraction may equal to the first best, or be distorted upward or downward. Indeed, in the first period, the regulator may be willing to increase the efficient firm's production above the first best to deter an inefficient firm from lying. This first-period overproduction also appears in Gaudet et al. (1995). How- 
ever, in their setting it comes from the resource constraint, while in our setting it only appears for a given set of parameters, and it is only explained by the ratchet effect. The regulator may also propose a pooling contract in which the extraction is set below the efficient firm's first-best level. Hence, if the stock is exhausted, total production is the same as with symmetric information but the extraction is distorted towards the future. This result differs from previous studies in which, an efficient firm never produces less than the first best during the first period. We also show that without commitment, the exhaustion of the resource may occur before or after the exhaustion with symmetric information. Finally, we find that when the discount factor is moderate, the ratchet effect implies that an increase in the discount factor does not necessarily reduce the first-period extraction which contradicts one of Hotelling's rule corollary.

Section 2 presents the model and assumptions. Section 3 characterizes the benchmarks, that is optimal contracts under symmetric information and under asymmetric information with commitment. In Section 4, the allocation under asymmetric information without commitment is defined. For each cases, we determine the extraction level and the tax revenue. To simplify the analysis, we assume that the resource is worthless at the end of the second period. Moreover, we focus on parameters that are such that with symmetric information it is optimal for each type to extract a positive quantity in the first period and to exhaust the stock during the second period. This restriction on parameters does not guarantee that under asymmetric information, it would be optimal to exhaust the resource over two periods, or to extract a positive quantity at each period. The results are discussed in Section 5. The proofs are relegated to an appendix at the end of the paper.

\section{General settings}

The regulator delegates to a firm the extraction of a resource available in quantity $S$. The extraction is denoted by $q$, and the extraction cost function is given by:

$c(\theta, q)=\theta q+\frac{b}{2} q^{2}$ 
The contract is a two-variable contract $C_{t}=\left(\tau_{t}, q_{t}\right)$. It specifies a tax $\tau_{t}$ and a quantity to extract $q_{t}$ at each period. We assume that the regulator is a price taker in the world market. Let us denote and assume that $X_{t} \equiv p_{t}-\theta>0$. This assumption ensures that under symmetric information, if the stock is not exhausted, the extraction is positive at each period.

During each period, the firm obtains the revenue net of extraction costs, and pays a tax to the regulator. Its payoff is given by:

$u\left(\theta, q_{t}, \tau_{t}, p_{t}\right)=p_{t} q_{t}-c\left(\theta, q_{t}\right)-\tau_{t}$

The regulator receives the tax and gets at each period:

$V_{R}=\tau_{t}$

The regulator and the firm have the same discount factor $\delta \in[0,1]$. Let us define $\tau_{t}^{R}$ the tax capturing the revenue net of the extraction costs. By definition $u\left(\theta, q_{t}, \tau_{t}^{R}, p_{t}\right)=0$. Hence,

$\tau_{t}^{R} \equiv p_{t} q_{t}-c\left(\theta, q_{t}\right)$

The periods are linked through the resource stock. The stock constraint is given by:

$\sum_{t=1}^{T} q_{t} \leq S$

$T$ is the terminal period, that is the period during which the contractual relationship between the regulator and the firm ends. Hence, $T$ is the number of contracting periods. At $t=T$, the stock is not necessarily exhausted. We assume that after the second period, the resource is worthless, hence $T \leq 2$. In section 5 , we discuss how the inability to commit affects the exhaustion date.

The following superscripts are used: $F B$ for symmetric information, $F C$ for asymmetric 
information with commitment, $N C$ for asymmetric information without commitment.

\section{Benchmarks}

\subsection{Symmetric information}

We assume that there are only two types of firms, efficient and inefficient $\theta=\{\underline{\theta}, \bar{\theta}\}$ and we use the following notation $\Delta \theta=\bar{\theta}-\underline{\theta}>0 .{ }^{5}$ We denote by $\underline{z}_{t}$ and $\bar{z}_{t}$, the variables $z$ in period $t$ that respectively refer to an efficient and an inefficient firm. The regulator maximizes its payoff subject to the firm's participation constraint and the stock constraint. The firm's outside opportunity is normalized to zero. The firm only participates if it receives a nonnegative payoff. The problem is the following:

$$
\begin{aligned}
\max _{\left\{q_{1}, \tau_{1}, q_{2}, \tau_{2}\right\}} V_{R}^{F B} & =\tau_{1}+\delta \tau_{2} \text { subject to } \\
& u\left(\theta, q_{1}, \tau_{1}, p_{1}\right)+\delta u\left(\theta, q_{2}, \tau_{2}, p_{2}\right) \geq 0 \\
& q_{1}+q_{2} \leq S
\end{aligned}
$$

At the optimum (6) binds, the regulator captures the entire rent and $\tau_{1}+\delta \tau_{2}=\tau_{1}^{R}+\delta \tau_{2}^{R}$. Using the binding constraint, the problem becomes: $\max _{\left\{q_{1}, q_{2}\right\}} V_{R}^{F B}=\tau_{1}^{R}+\delta \tau_{2}^{R}$ subject to (7). If (7) is slack, the production at each period is:

$q_{t}^{F B}=\frac{X_{t}}{b}$

From $\underline{q}_{t}^{F B}>\bar{q}_{t}^{F B}$, none of the firms exhaust the stock if:

$S>\frac{\bar{X}_{1}}{b}+\frac{\bar{X}_{2}}{b}=S^{a}$

\footnotetext{
${ }^{5}$ By considering only two types, we depart from Gaudet et al. (1995), Osmundsen (1998) and Hung et al. (2006). However, with a continuum of types, as shown by Laffont and Tirole (1988), without commitment, full-separating contracts are not optimal, and some amount of pooling should be implemented.
} 
If $S<S^{a}$, using the subscript $e$ for exhausted stock, the production is:

$$
\begin{aligned}
& q_{1_{e}}^{F B}=\frac{X_{1}}{b}-\frac{\delta\left(X_{1}+X_{2}-b S\right)}{b(1+\delta)} \\
& q_{2_{e}}^{F B}=\frac{X_{2}}{b}-\frac{\left(X_{1}+X_{2}-b S\right)}{b(1+\delta)}
\end{aligned}
$$

In Appendix A, we define two assumptions. $A 1_{a}$ ensures that $\bar{q}_{1_{e}}^{F B}>0$ (which also implies $\underline{q}_{1_{e}}^{F B}>0$ ), and $A 1_{b}$ ensures that $\underline{q}_{2_{e}}^{F B}>0$ (which also implies $\bar{q}_{2_{e}}^{F B}>0$ ).

Under symmetric information, the regulator captures the entire revenue and if $S<S^{a}$, the extraction path satisfies the Hotelling's rule, the marginal net benefit grows over time at the rate of discount. An increase in the discount factor decreases the first-period extraction.

From this point on we assume that the parameters are such that $S<S^{a}$ and that $A 1_{a}$ and $A 1_{b}$ are satisfied, meaning that under symmetric information, both types exhaust the stock and extract a positive quantity at each period. Hence, in what follows, when we refer to the first-best levels, we refer to the extractions defined by (10) and (11). We also assume that the regulator does not know the efficiency parameter $\theta$. The firm may be efficient $(\underline{\theta})$ with probability $\nu_{1}$ or inefficient $(\bar{\theta})$ with the complementary probability.

\subsection{Asymmetric information with commitment}

The regulator commits during the first period to the second-period contracts and proposes a menu of two contracts specifying the productions $q_{t}$ and the tax $\tau_{t}$. The regulator maximizes its payoff subject to the stock constraints and to the firms' incentive and participation constraints. 
The regulator's problem is as follows:

$$
\begin{aligned}
& \max _{\left\{\underline{q}_{1}, \underline{\tau}_{1}, \underline{q}_{2}, \underline{\tau}_{2}, \bar{q}_{1}, \bar{\tau}_{1}, \bar{q}_{2}, \bar{\tau}_{2}\right\}} V_{R}^{F C}=\nu_{1}\left(\underline{\tau}_{1}+\delta \underline{\tau}_{2}\right)+\left(1-\nu_{1}\right)\left(\bar{\tau}_{1}+\delta \bar{\tau}_{2}\right) \text { subject to } \\
& u\left(\underline{\theta}, \underline{q}_{1}, \underline{\tau}_{1}, p_{1}\right)+\delta u\left(\underline{\theta}, \underline{q}_{2}, \underline{\tau}_{2}, p_{2}\right) \geq 0 \\
& u\left(\bar{\theta}, \bar{q}_{1}, \bar{\tau}_{1}, p_{1}\right)+\delta u\left(\bar{\theta}, \bar{q}_{2}, \bar{\tau}_{2}, p_{2}\right) \geq 0 \\
& u\left(\underline{\theta}, \underline{q}_{1}, \underline{\tau}_{1}, p_{1}\right)+\delta u\left(\underline{\theta}, \underline{q}_{2}, \underline{\tau}_{2}, p_{2}\right) \geq u\left(\underline{\theta}, \bar{q}_{1}, \bar{\tau}_{1}, p_{1}\right)+\delta u\left(\underline{\theta}, \bar{q}_{2}, \bar{\tau}_{2}, p_{2}\right) \\
& u\left(\bar{\theta}, \bar{q}_{1}, \bar{\tau}_{1}, p_{1}\right)+\delta u\left(\bar{\theta}, \bar{q}_{2}, \bar{\tau}_{2}, p_{2}\right) \geq u\left(\bar{\theta}, \underline{q}_{1}, \underline{\tau}_{1}, p_{1}\right)+\delta u\left(\bar{\theta}, \underline{q}_{2}, \underline{\tau}_{2}, p_{2}\right) \\
& \underline{q}_{1}+\underline{q}_{2} \leq S \\
& \bar{q}_{1}+\bar{q}_{2} \leq S
\end{aligned}
$$

At the optimum (14) and (15) bind. From (14) binding, $\bar{\tau}_{1}^{F C}+\delta \bar{\tau}_{2}^{F C}=\bar{\tau}_{1}^{R}+\delta \bar{\tau}_{2}^{R}$, and by combining the two binding constraints: $\underline{\tau}_{1}^{F C}+\delta \underline{\tau}_{2}^{F C}=\underline{\tau}_{1}^{R}+\delta \underline{\tau}_{2}^{R}-\Delta \theta\left(\bar{q}_{1}+\delta \bar{q}_{2}\right)$.

The non-binding constraint (13) becomes: $\Delta \theta\left(\bar{q}_{1}+\delta \bar{q}_{2}\right) \geq 0$ and is always satisfied, while (16) becomes: $\Delta \theta\left(\underline{q}_{1}-\bar{q}_{1}+\delta\left(\underline{q}_{2}-\bar{q}_{2}\right)\right) \geq 0$. By replacing taxes into (12), the problem becomes:

$\max _{\left\{\underline{q}_{1}, \bar{q}_{1}, \underline{q}_{2}, \bar{q}_{2}\right\}} V_{R}^{F C}=\nu_{1}\left(\underline{\tau}_{1}^{R}-\Delta \theta \bar{q}_{1}+\delta\left(\underline{\tau}_{2}^{R}-\Delta \theta \bar{q}_{2}\right)\right)+\left(1-\nu_{1}\right)\left(\bar{\tau}_{1}^{R}+\delta \bar{\tau}_{2}^{R}\right)$

subject to $(16),(17),(18)$

By solving the problem, we can easily show that an efficient firm extracts the first best during each period (no distortion at the top), and gets an informational rent. This informational rent reduces the tax collected from an efficient firm.

If (18) is slack, an inefficient firm produces at each period:

$$
\begin{aligned}
& \bar{q}_{1}^{F C}=\frac{\bar{X}_{1}}{b}-\frac{\Delta \theta \nu_{1}}{b\left(1-\nu_{1}\right)}=\bar{q}_{1 e}^{F B}+\frac{\delta\left(\bar{X}_{1}+\bar{X}_{2}-b S\right)}{b(1+\delta)}-\frac{\Delta \theta \nu_{1}}{b\left(1-\nu_{1}\right)} \\
& \bar{q}_{2}^{F C}=\frac{\bar{X}_{2}}{b}-\frac{\Delta \theta \nu_{1}}{b\left(1-\nu_{1}\right)}=\bar{q}_{2 e}^{F B}+\frac{\bar{X}_{1}+\bar{X}_{2}-b S}{b(1+\delta)}-\frac{\Delta \theta \nu_{1}}{b\left(1-\nu_{1}\right)}
\end{aligned}
$$


The extraction defined above is optimal if:

$S>\frac{\bar{X}_{1}}{b}+\frac{\bar{X}_{2}}{b}-\frac{2 \Delta \theta \nu_{1}}{b\left(1-\nu_{1}\right)}=S^{b}$, with $S^{b}<S^{a}$

If $S>S^{b}$ and if assumptions $A 2_{a}$ and $A 2_{b}$ defined in Appendix A are satisfied (i.e, if $\bar{q}_{1}^{F C}>0$ and $\bar{q}_{2}^{F C}>0$ ), an inefficient firm does not exhaust the stock. During the first period, it extracts a lower quantity than the first best, the latter being defined by (10). Hence, the first-period tax revenue is lower than the first best. During the second period, an inefficient firm may produce more or less than the first best, the latter being defined by (11). Hence, the tax revenue at this period can be greater than the first best. Nevertheless, total production of an inefficient firm is reduced, and the discounted tax revenue is lower than the first best.

If $S<S^{b}$, an inefficient firm produces:

$\bar{q}_{1_{e}}^{F C}=\bar{q}_{1_{e}}^{F B}-\frac{\Delta \theta \nu_{1}(1-\delta)}{b\left(1-\nu_{1}\right)(1+\delta)}=\bar{q}_{1}^{F C}-\frac{\delta\left(\left(1-\nu_{1}\right)\left(\bar{X}_{1}+\bar{X}_{2}-b S\right)-2 \Delta \theta \nu_{1}\right)}{b\left(1-\nu_{1}\right)(1+\delta)}$
$\bar{q}_{2_{e}}^{F C}=\bar{q}_{2_{e}}^{F B}+\frac{\Delta \theta \nu_{1}(1-\delta)}{b\left(1-\nu_{1}\right)(1+\delta)}=\bar{q}_{2}^{F C}-\frac{\left(1-\nu_{1}\right)\left(\bar{X}_{1}+\bar{X}_{2}-b S\right)-2 \Delta \theta \nu_{1}}{b\left(1-\nu_{1}\right)(1+\delta)}$

If $S<S^{b}$ and if assumption $A 2_{c}$ defined in Appendix A is satisfied (i.e, if $\bar{q}_{1_{e}}^{F C}>0$ ), it is optimal for an inefficient firm to exhaust the stock, and the regulator cannot decrease extraction during one period without increasing extraction during the other period. As a result, it is optimal to decrease the informational rent and to decrease the inefficient firm's extraction at the most valued period, that is, the first period (from $\delta<1$ ). Hence, during the first period, the inefficient firm's production is distorted downward, while during the second period it is distorted upward. As compared to the first best, total production is the same, but the extraction is shifted towards the future. As a result, during the first period the revenue tax collected from an inefficient firm is lower, while during the second period the tax revenue is higher. As compared to an efficient firm, an inefficient firm does not receive a rent but the tax revenue collected is also lower than the first best since the extraction is distorted. 
The intuition behind the inefficient firm's second-period overproduction is the same as in Hung et al. (2006). The first-period informational rent decreases the inefficient firm's firstperiod production which relaxes the stock constraint and allows for a higher extraction during the second period. The Hotelling's rule for an inefficient firm is modified to take into account the informational rent. An increase in the discount factor decreases the first-period extraction. Regardless of the size of the stock, we show in Appendix A that (16) is always satisfied. Hence, there are no countervailing incentives, only an efficient firm misrepresents its type.

Note that the assumptions on the positivity of an inefficient firm's extraction $\left(A 2_{a}, A 2_{b}\right.$ and $A 2_{c}$ ) imply that it is not optimal to exclude an inefficient firm. The exclusion is optimal when the size of the information asymmetry and the probability to face an efficient firm are relatively high. In this case, the informational rent is significant and the probability to face an inefficient firm is so low that the regulator prefers to exclude an inefficient firm. The tax revenue when there is exclusion is defined in the Appendix A.

\section{Asymmetric information without commitment}

When the regulator is unable to commit during the first period to the second-period contracts, the firm chooses a contract during the first period and the regulator updates its belief accordingly. In the second period, the regulator proposes one contract for each type, or captures all the revenue if it knows the firm's type. The regulator updates its prior belief $\left(\nu_{1}\right)$ according to a Bayesian rule. The posterior beliefs $\nu_{2}=\left\{\underline{\nu}_{2}, \bar{\nu}_{2}\right\}$ are:

$$
\underline{\nu}_{2}=\frac{x \nu_{1}}{x \nu_{1}+y\left(1-\nu_{1}\right)} \text { and } \quad \bar{\nu}_{2}=\frac{(1-x) \nu_{1}}{(1-x) \nu_{1}+(1-y)\left(1-\nu_{1}\right)} \quad \text { with } x \in[0,1] \text { and } y \in[x, 1]
$$

Let us denote $\underline{C}_{t}=\left(\underline{\tau}_{t}, \underline{q}_{t}\right)$, the contract designed for an efficient firm and $\bar{C}_{t}=\left(\bar{\tau}_{t}, \bar{q}_{t}\right)$ the contract designed for an inefficient firm.

$x$ is the probability that an efficient firm chooses $\underline{C}_{1}$ in the first period.

$y$ is the probability that an inefficient firm chooses $\underline{C}_{1}$ in the first period.

$\underline{\nu}_{2}$ is the probability that the firm is efficient knowing that $\underline{C}_{1}$ is chosen.

$\bar{\nu}_{2}$ is the probability that the firm is efficient knowing that $\bar{C}_{1}$ is chosen. 
Without loss of generality, we assume that $x>y$.

To simplify the notation, we denote by:

- $z_{2} \underline{C}_{1}$, the second-period variable $z$ if $\underline{C}_{1}$ has been chosen (e.g, if $\underline{C}_{1}$ is chosen, the efficient firm's second-period tax is denoted by $\left.\underline{\tau}_{2 / \underline{C}_{1}}\right)$.

- $z_{2 / \bar{C}_{1}}$, the second-period variable $z$ if $\bar{C}_{1}$ has been chosen (e.g, if $\bar{C}_{1}$ is chosen, the inefficient firm's second-period extraction is denoted by $\left.\bar{q}_{2 / \bar{C}_{1}}\right)$.

The regulator's payoff is the expected sum of taxes:

$$
\begin{aligned}
V_{R}^{N C} & =\left(\nu_{1} x+\left(1-\nu_{1}\right) y\right)\left(\underline{\tau}_{1}+\delta\left(\underline{\nu}_{2} \underline{\tau}_{2 / \underline{C}_{1}}+\left(1-\underline{\nu}_{2}\right) \bar{\tau}_{2 / \underline{C}_{1}}\right)\right) \\
& +\left(\nu_{1}(1-x)+\left(1-\nu_{1}\right)(1-y)\right)\left(\bar{\tau}_{1}+\delta\left(\bar{\nu}_{2} \underline{\tau}_{2 / \bar{C}_{1}}+\left(1-\bar{\nu}_{2}\right) \bar{\tau}_{2 / \bar{C}_{1}}\right)\right)
\end{aligned}
$$

When the regulator is unable to commit, implementing full-separating contracts is not always optimal. Semi-separating contracts, or a pooling contract may also be implemented. The values of $x$ and $y$ determine the type of contracts implemented during the first period. Under full separation $(x, y)=(1,0)$, each firm chooses the contract designed for its type. The regulator identifies the type of firm it is dealing with. During the second period, $\underline{\nu}_{2}=1-\bar{\nu}_{2}=1$, symmetric information occurs, the regulator captures all of the revenue and $\tau_{2}=\tau_{2}^{R}$. Under semi separation, one type of firm plays a pure strategy, while the other plays a mixed strategy: $(x, y)=(1, y)$ or $(x, 0)$. If during the first period, the contract chosen is designed for the type that plays a mixed strategy, then the regulator infers the firm's type and $\tau_{2}=\tau_{2}^{R}$. If not, information asymmetry still occurs. Under a pooling contract, during the second period, no updating is possible since only one contract is proposed, $\underline{\nu}_{2}=\bar{\nu}_{2}=\nu_{1}$.

We use perfect Bayesian equilibrium as our solution concept. The problem is solved backwards, for a given stock $S_{2}$ and updated beliefs $\nu_{2}$, the second-period contracts are similar to the ones with commitment. Indeed, with commitment the allocation is the same as the repetition of the optimal static contracts. If during the second period, there is still informa- 
tion asymmetry, an efficient firm gets an informational rent $\Delta \theta \bar{q}_{2}$ and produces $\underline{q}_{2}^{F B}$ (or the remaining stock), while an inefficient firm gets no rent and produces $\bar{q}_{2}^{F C}$ (or the remaining stock). These contracts are defined in Appendix B. First-period decisions affect the secondperiod extraction and the second-period informational rent through the updated beliefs and the remaining stock. The first-period participation and incentive constraints denoted by $\underline{P C}$, $\overline{P C}, \underline{I C}$ and $\overline{I C}$ are:

$u\left(\underline{\theta}, \underline{q}_{1}, \underline{\tau}_{1}, p_{1}\right)+\delta \Delta \theta \bar{q}_{2 / \underline{C}_{1}} \geq 0$

$u\left(\bar{\theta}, \bar{q}_{1}, \bar{\tau}_{1}, p_{1}\right) \geq 0$

$u\left(\underline{\theta}, \underline{q}_{1}, \underline{\tau}_{1}, p_{1}\right)+\delta \Delta \theta \bar{q}_{2 / \underline{C}_{1}} \geq u\left(\underline{\theta}, \bar{q}_{1}, \bar{\tau}_{1}, p_{1}\right)+\delta \Delta \theta \bar{q}_{2 / \bar{C}_{1}}$

$u\left(\bar{\theta}, \bar{q}_{1}, \bar{\tau}_{1}, p_{1}\right) \geq u\left(\bar{\theta}, \underline{q}_{1}, \underline{\tau}_{1}, p_{1}\right)$

The stock constraints denoted by $S C$ are:

$\underline{q}_{1}+\underline{q}_{2 / \underline{C}_{1}} \leq S$

$\bar{q}_{1}+\bar{q}_{2 / \bar{C}_{1}} \leq S$

$\bar{q}_{1}+\underline{q}_{2 / \bar{C}_{1}} \leq S$

$\underline{q}_{1}+\bar{q}_{2 / \underline{C}_{1}} \leq S$

As usual, $(\overline{P C})$ and $(\underline{\mathrm{IC}})$ bind, which implies that $(\underline{\mathrm{PC}})$ is satisfied. First, we study the case in which only $(\underline{\mathrm{IC}})$ binds. However, if the parameters are such that $(\overline{I C})$ is not satisfied, both incentive constraints must bind.

\subsection{Only the efficient firm's incentive constraint binds}

If only ( $\underline{\mathrm{IC}})$ binds, assuming that $(\overline{I C})$ is satisfied, we show in Appendix $\mathrm{C}$, that it is optimal to propose during the first period full-separating contracts $(x=1$ and $y=0)$. Such contracts imply that during the second period, the regulator is able to capture the entire revenue. 
Under full separation, an efficient firm produces the first best, and an inefficient firm may not exhaust the stock. If so, it produces $\bar{q}_{1}^{F C}$ given by (20) and $\bar{q}_{2}^{F B}$ given by (8). These full-separating contracts are implemented if:

$S>\frac{\bar{X}_{1}}{b}+\frac{\bar{X}_{2}}{b}-\frac{\Delta \theta \nu_{1}}{b\left(1-\nu_{1}\right)}=S^{c}$, with $S^{b}<S^{c}<S^{a}$

$S^{c}>S^{b}$, since without commitment, full separation implies that the inefficient firm's second-period production is not distorted by the informational rent. If $S<S^{c}$, an inefficient firm produces during the first period $\bar{q}_{1_{e}}^{F C}$ defined by (23), and exhausts the stock during the second period. For both types, the extraction path is the same as with commitment.

We now determine the conditions under which $(\overline{I C})$ is satisfied. An inefficient firm reveals its type only if the cost of lying exceeds the gains. Indeed, when an inefficient firm misrepresents its type during the first period, it overproduces and bears the costs $\Delta \theta \underline{q}_{1}$ but captures the informational rent: $\Delta \theta\left(\bar{q}_{1}+\delta \bar{q}_{2}\right)$. During the second period, the tax will be $\underline{\tau}_{2}^{R}$, an inefficient firm prefers to leave the relationship rather than to bear the overproduction costs $\Delta \theta \underline{q}_{2}$. The condition on the parameters to ensure that $(\overline{I C})$ is satisfied depends on the stock constraints:

$$
\begin{aligned}
& \left(1-\delta+2 \delta \nu_{1}\right) \Delta \theta-\delta\left(1-\nu_{1}\right)\left(\bar{X}_{1}+(2+\delta) \bar{X}_{2}-b S\right) \geq 0 \\
& (1-\delta)\left(1-\delta \nu_{1}\right) \Delta \theta-\delta\left(1-\nu_{1}\right)\left(\delta \bar{X}_{2}-\bar{X}_{1}+b S\right) \geq 0
\end{aligned}
$$

Assuming that $S>S^{c}$, and that $A 2_{a}$ is satisfied, $(\overline{I C})$ is satisfied only if $A 3_{a}$ is satisfied. Assuming that $S<S^{c}$, and that $A 2_{c}$ is satisfied, $(\overline{I C})$ is satisfied only if $A 3_{b}$ is satisfied. By studying $A 3_{a}$ and $A 3_{b}$, an inefficient firm understates its costs during the first period when the second-period price and the discount factor are high, that is when the informational rent related to the second period is significant. It also misrepresents its type when the probability of facing an efficient firm is low, that is, when the trade-off between informational rent and productive efficiency induces a relatively high informational rent. Finally, the size of the information asymmetry $(\Delta \theta)$ increases both the informational rent and the costs of lying. Never- 
theless, an inefficient firm has a greater incentive to lie when the information asymmetry is low.

The following proposition describes the optimal contracts when only $(\overline{P C})$ and $(\underline{\text { IC }})$ bind.

Proposition 1 Under asymmetric information without commitment, during the first period, if $A 3_{a}$ or $A 3_{b}$ is satisfied, there are no countervailing incentives, and full-separating contracts are implemented.

- An efficient firm produces the first best at each period, and receives an informational rent. This rent is given during the first period.

- If $S^{c}<S<S^{a}$, and if $A 2_{a}$ and $A 3_{a}$ are satisfied, an inefficient does not exhaust the stock. It produces during the first period the same as with commitment, and during the second period, it produces more. The tax revenue is lower than with commitment.

- If $S^{b}<S<S^{c}$, and if $A 2_{c}$ and $A 3_{b}$ are satisfied, an inefficient exhausts the stock and produces at each period a higher amount than with commitment. The tax revenue is lower than with commitment.

- If $S<S^{b}$, and if $A 2_{c}$ and $A 3_{b}$ are satisfied, an inefficient exhausts the stock and produces at each period the same as with commitment. The tax revenue is the same with and without commitment. However, without commitment the informational rent must be given during the first period, while with commitment the timing of the payment is not constrained.

Conditions $A 3_{a}$ and $A 3_{b}$ are defined above in this section, condition $A 2_{a}$ and $A 2_{c}$ are defined in Appendix A. See proof in Appendix C.

As compared to the first best, the regulator collects less tax revenue from an efficient firm due to the informational rent, and less tax revenue from an inefficient firm due to the productive distortion.

If $S>S^{b}$, during the first period, an inefficient firm produces at least the same as with commitment, and during the second period, it produces more. The higher production implies 
higher tax revenue collected from an inefficient firm but also higher informational rent. The informational rent effect dominates and the tax revenue is lower without commitment than with commitment.

Nevertheless, if $S<S^{b}$, the extraction is the same with and without commitment, and the regulator collects exactly the same tax revenue. During the second period, the regulator captures the entire revenue. Hence, if the stock is sufficiently low to be exhausted with commitment, and if the discount factor is sufficiently low to avoid countervailing incentives, the inability to commit does not affect the extraction path nor the expected tax revenue. The stock constraint implies that there is no increase in the inefficient firm's second-period production that would increase the informational rent. The inability to commit only affects the timing at which taxes from an efficient firm are collected. If the regulator is able to commit, it gives to an efficient firm $\Delta \theta \bar{q}_{t}$ at each period, or it gives $\Delta \theta\left(\bar{q}_{1}+\delta \bar{q}_{2}\right)$ during the first period, the two options are equivalent in our setting. On the opposite, without commitment, the informational rent $\Delta \theta\left(\bar{q}_{1}+\delta \bar{q}_{2}\right)$ must be given during the first period which implies that the regulator must have enough cash to pay the firm.

\subsection{Both incentive constraints bind}

$(\overline{P C})$ binding implies $\bar{\tau}_{1}^{N C}=\bar{\tau}_{1}^{R}$, and $(\overline{P C})$ and $(\underline{\mathrm{IC}})$ binding imply that:

$\underline{\tau}_{1}^{N C}=\underline{\tau}_{1}^{R}-\Delta \theta\left(\bar{q}_{1}+\delta \bar{q}_{2 / \bar{C}_{1}}-\delta \bar{q}_{2 / \underline{C}_{1}}\right)$

During the first period, an efficient firm receives an informational rent: $\Delta \theta\left(\bar{q}_{1}+\delta \bar{q}_{2 / \bar{C}_{1}}\right)$. From $(\overline{P C})$ and $(\underline{\mathrm{IC}})$ binding, $(\underline{\mathrm{IC}})$ binding implies that:

$\Delta \theta \underline{q}_{1}=\Delta \theta\left(\bar{q}_{1}+\delta \bar{q}_{2 / \bar{C}_{1}}-\delta \bar{q}_{2 / \underline{C}_{1}}\right) \Leftrightarrow \underline{q}_{1}=\bar{q}_{1}+\delta\left(\bar{q}_{2 / \bar{C}_{1}}-\bar{q}_{2 / \underline{C}_{1}}\right)$

The left-hand side represents the costs of lying (overproduction), while the right-hand side represents the gains from lying (informational rent). When both incentive constraints bind, $\underline{q}_{1}$ and $\bar{q}_{1}$ are positively correlated. If $\bar{q}_{1}$ increases, the informational rent increases and $\underline{q}_{1}$ must 
increase as well to compensate the cost of lying.

If $S<S^{a}$, we show in Appendix F that an efficient firm always exhausts the stock during the second period. Of the two main cases, the one in which only an efficient exhausts the stock, the other in which both firms exhaust the stock, only the latter is presented in the main text. ${ }^{6}$

In Appendix E.1, we determine for each contract (full-separating and pooling contracts) the extraction path when both types exhaust the stock, and in Appendix E.2, we define the tax revenue generated by each contract to determine the conditions under which one contract is preferred to the other. We define in Appendix E.2 the threshold on the discount factor $\delta_{2}$ above which a pooling contract is optimal.

If the stock is sufficiently low to be exhausted by both firms and if both incentive constraints bind, the first-period full-separating contracts denoted by the upper-script $F S$ are such that an efficient produces more than the first best $\left(\underline{q}_{1_{e}}^{F S}>\underline{q}_{1_{e}}^{F B}\right)$, while and inefficient firm produces less than the first best $\left(\bar{q}_{1_{e}}^{F S}<\bar{q}_{1_{e}}^{F B}\right)$, and even less than with commitment $\left(\bar{q}_{1_{e}}^{F S}<\bar{q}_{1}^{F C}<\bar{q}_{1_{e}}^{F C}\right)$. By contrast, the optimal first-period pooling contract is such that both firms produce the inefficient firm's first-best level $\left(\bar{q}_{t_{e}}^{F B}\right)$. Hence, during the first period, an efficient firm produces a lower quantity than the first best, and in the second period, a larger quantity than the first best. As compared to full-separating contracts, an efficient firm's first-period extraction is now distorted downward, while an inefficient produces at each period the same as under symmetric information.

The following proposition summarizes the optimal contracts when both firms exhaust the stock, and when both incentive constraints bind:

Proposition 2 Under asymmetric information without commitment, if the stock is relatively low (the conditions are defined in Appendix E.1), and if $A 3_{b}$ does not hold, there exists a

\footnotetext{
${ }^{6}$ The former case is treated in Appendix D. The thresholds on the stock that are such that both firms exhaust the stock are also defined in Appendix D. We ignore cases in which an inefficient firm exhausts the stock if it chooses one contract and does not exhaust the stock when its chooses the other contract.
} 
threshold value of the discount factor $\delta_{2}$, defined by (58) in Appendix D, such that

- if $\delta<\delta_{2}$, full-separating contracts are implemented and during the first period,

- an efficient firm produces more than under symmetric information, while an inefficient firm produces less than with commitment. Both types of firms exhaust the stock during the second period.

- the modified Hotelling's rule is such that an increase in the discount factor does not necessarily reduce the first-period extraction.

- if $\delta>\delta_{2}$, during the first period, a pooling contract is implemented. Both firms produce the inefficient firm's first-best level and exhaust the stock during the second period.

For the proof see Appendix D.

As compared to with commitment, or to the case in which only $\underline{I C}$ binds, there is a distortion at the top. Proposition 2 shows that under full-separating contracts, if the stock is sufficiently low to be exhausted by both types, an efficient firm should produce more than the first best. This result holds even if none of the firms exhaust the stock (Appendix F) or if only an efficient firm exhausts the stock (Appendix D). Hence, this overproduction comes from the ratchet effect and not from the stock constraint. Nevertheless, the constraint implies that the efficient firm's second-period production is distorted while it is not without it. More specifically, it implies that the efficient firm's second-period production is lower than the first best, the latter being already too low due to the stock constraint. The efficient firm's firstperiod overproduction aims at preventing an inefficient firm from understating its costs. This overproduction also appears in Gaudet et al. (1995), however, in their setting it is explained by the stock constraint and by the assumption that the cost parameter changes at each period. Indeed, since an inefficient firm produces at each period less than the first best, the efficient firm's opportunity costs of extracting during the first period is lower. Taking into consideration that an efficient firm during the first period can be inefficient during the second period, it is optimal to set its first-period production above the first best. Hence, in Gaudet et al. (1995), this overproduction does not occur when the stock is not exhausted. 
Proposition 2 also states that under full-separating contracts, if the stock is sufficiently low to be exhausted by both types, an inefficient firm produces in the first period even less than with commitment. This result holds even if none of the firms exhaust the stock (Appendix F) or if only an efficient firm exhausts the stock (Appendix D). As with commitment the inefficient firm's first-period extraction is distorted downward to decrease the informational rent. However, the correlation of the productions induced by countervailing incentives calls for an even greater distortion. Indeed, if the inefficient firm's production is set to the full-commitment level, the efficient firm's production will be too high. During the second period, an inefficient firm produces more than with commitment and even more than the first best. Indeed, the first-period downward distortion relaxes the stock constraint. As compared to the full commitment case, or to the case without commitment and without countervailing incentives, the extraction is more distorted towards the future. From $\delta<1$, this implies that the discounted informational rent given to an efficient firm is lower than with commitment. Nevertheless, this lower rent must be given during the first period, while with commitment the regulator can distribute the rent over the two periods. Despite this lower rent, due to the efficient firm's productive distortion, and to the inefficient firm's greater productive distortion, the tax collected without commitment is lower than with commitment. Note that the inability to commit when there are countervailing incentives and full separation has an ambiguous effect on the inefficient firm's total production. Indeed, the first period-extraction is lower than with commitment but the second-period extraction is higher. Hence, total production can be greater or smaller with commitment. In other words, it may be optimal for an inefficient firm to exhaust the stock with commitment, while it is not optimal without commitment. The opposite also holds.

Proposition 2 also states that with full separation the modified Hotelling's rule is such that an increase in the discount factor may decrease the firms' second-period extraction. Indeed, with asymmetric information without commitment, the discount factor affects the production through three effects: a resource, an adverse selection, and a countervailing effects. The stock constraint implies that as the discount factor increases it is optimal to postpone extraction and to increase the second-period extraction, this is a direct implication of the Hotelling's rule. 
Moreover, an increase in the discount factor also increases the informational rent related to the second period. To compensate the regulator is willing to decrease the inefficient firm's second-period extraction. This effect is related to the adverse selection and also appears with commitment. Finally, due to countervailing incentives, the extraction is such that the cost of lying $\Delta \theta \underline{q}_{1}$ must equal to the informational rent $\Delta \theta\left(\bar{q}_{1}+\delta\left(S-\bar{q}_{1}\right)\right)$. Hence, as the discount factor increases, the informational rent related to the second period increases and to compensate the regulator increases the cost of lying that is the efficient firm's first-period production. For an inefficient firm, the countervailing effect goes in the opposite direction, the regulator decreases the first-period extraction (and the first-period informational rent) to compensate. As the discount factor increases, the countervailing effect decreases the efficient firm's secondperiod extraction, while the resource effect increases the extraction. For an inefficient firm, as the discount factor increases, the resource and the countervailing effects increase the secondperiod production, while the adverse selection effect decreases this production.

If the discount factor is high, and if the stock is sufficiently low to be exhausted by both types, the regulator implements during the first period a pooling contract. This pooling contract is such that both firms produce the inefficient firm's first-best level. The decrease in production required to decrease the second-period informational rent perfectly offsets the increase in production required to take into account that an efficient firm also chooses this contract. As a result, both firms produce the inefficient firm's first-best level. Under this pooling contract, the discounted tax revenue collected from an inefficient firm is the same as the first best. However, due to the efficient firm's productive distortion and to the second-period informational rent, the discounted tax revenue collected from an efficient firm is lower. Moreover, the tax revenue is lower under a pooling contract than with commitment. Note that if only an efficient firm exhausts the stock, the pooling contract would be such that the first-period production lies between first-best level of each type. 


\section{Concluding remarks}

In this last section, we discuss the implementation of the contracts using real world resource exploitation contracts, and we explain how our results are affected if a third period is considered. Finally, we present two directions for future research.

This paper determines the optimal contracts when the regulator cannot commit to longterm contracts and under asymmetric information on costs. We found that optimal contracts mainly depend on two parameters, the size of stock and the discount factor. The large variety of contracts defined in the paper can be surprising, but it may in fact reflect the diversity of contracts observed in the real world. In the paper, we assume that the regulator proposes a contract specifying a production level and a tax (lump sum payment). However, the secondbest solutions can be implemented using different contractual forms. In this discussion, we focus on three instruments, a royalty to induce the production distortion desired, a license fee to capture the revenue, and an upper bound or a lower bound on the production. The upper bound aims at decreasing the efficient firm's informational rent, while the lower bound aims at keeping the inefficient firm's rent to zero. This production restriction is not unusual since sliding scale royalties are often used. In this discussion, we focus on the case in which the stock is relatively low, and so, when it is always optimal for both firms to exhaust the stock.

First, it is interesting to see how the shape of the contract is affected by the inability to commit. Let us compare the optimal contracts with and without commitment when the discount factor is low since they induce the same outcome (no countervailing incentives). Regardless of the ability to commit, the first-period contract designed for an inefficient firm implies a royalty, a license fee and an upper limit to the production. However, without commitment the second-period contract has no royalty, while with commitment there is one. Moreover, the first-period royalty should be lower without commitment. Indeed, the production targeted is the same in both cases, but without commitment since there is no royalty during the second period, the opportunity cost of extracting during the first period is higher. Hence, to induce the same extraction level, the first-period royalty must be lower without commitment. Finally, the 
license fee should be such that the government is able to capture the entire revenue. Regardless of the ability to commit, the contract designed for an efficient firm only specifies a license fee and a lower bound on the production, but no royalties (high-powered incentive scheme). The inability to commit implies that the efficient firm's informational rent must be given upfront. As a result, without commitment an efficient firm gets a positive profit only during the first period, and no profit during the second period. With commitment, the discounted sum of profit is the same but the discounted profit can be split between the two periods.

Second, if the discount factor is relatively low (countervailing incentives and full separation), the inefficient firm's first-period contract implies a royalty that can be lower or higher than with commitment. Two opposite effects are at stake. Without commitment, the extraction should be further distorted towards the future, which implies a higher royalty, however the absence of second-period royalty increases the opportunity cost of extracting during the first period, which implies a lower royalty. On the opposite, the first-period contract designed for an efficient firm specifies a license fee, and a negative royalty. This negative royalty means that a firm will receive a payment for each unit produced, it does not mean that the discounted tax revenue is negative since the regulator also receives a license fee. This negative tax rate is not unusual especially during the first period of the extraction.

Third, if the discount factor is large, the regulator proposes during the first period only one contract that only specifies a license fee and an upper limit for the production. Indeed, both firms would like to produce more than the regulator's desired level $\left(\bar{q}_{1_{e}}^{F B}\right)$. An efficient firm because it is more efficient, and an inefficient because it will have to pay a royalty during the second period.

In this discussion, we have focused on a royalty and a license fee. However, let us briefly discuss production sharing agreements (PSA). In its most simple form, a PSA specifies a share of the revenue that could be allocated to the cost reimbursement. The payment received for the cost reimbursement is called the cost oil. Depending on the share allocated to the cost reimbursement, costs can be partly or entirely reimbursed. The contract should also specify the share of the profit oil received by the firm, the profit oil being the remaining revenue after 
the cost oil payment. First, if the government wants to avoid productive distortion, then the share allocated to the cost reimbursement should be high enough for the costs to be entirely reimbursed. In this case, the share of the profit oil will be similar to a profit tax, and the regulator should set this profit tax such that it captures the entire revenue, or such that it leaves the firm with an informational rent. Second, if the government wants to distort the production, then, the share of the revenue allocated to the costs and the share of the profit oil are substitute instruments. More specifically, the PSA is similar to a royalty rate. However, in this case the government needs an additional instrument, such as a license fee, to capture the tax revenue. Finally, as with a royalty and a license fee, the PSA should also specify a lower or an upper bound on production. This production bound does not affect the firm's extraction if it chooses the contract designed for its type, but it restricts the extraction of a firm that would choose the contract designed for the other type.

This paper has shown that if the parameters are such that it is optimal for both firms to exhaust the stock, and if the discount factor is sufficiently low (no countervailing incentives), the tax revenue and the extraction path are the same with and without commitment, and thus the inability to commit is costless. This inability only constrained the timing at which taxes are collected. If the terminal period is greater than two, separating firms at the period before exhaustion is always optimal and costless. Nevertheless, in the previous periods, the inability to commit is costly. Hence, if the discount factor is low, the exhaustion of the resource decreases the cost of non-commitment and this cost is null in a two-period model. In this paper, we have assumed that the resource is worthless after the second period. Therefore, the contractual relationship may at most last two periods. By considering that resource extraction can take place over up to three periods, we can discuss how the inability to commit affects the terminal period and the exhaustion date (see Appendix G.1). Under asymmetric information without commitment, if the discount factor is relatively large, then there is a countervailing incentive and in the first period, full-separating contracts are implemented. If so, an efficient firm may exhaust the resource before the first best, and an inefficient firm may exhaust the resource after the first best. On the opposite, if the discount factor is large, then, in the first period, a pooling 
contract is implemented. An efficient firm may exhaust the stock at a later period, while the inefficient firm's extraction is the same as under symmetric information. Note that as the number of contracting periods $T$ increases, the inefficient firm's incentive constraint is less likely to be satisfied, and both incentive constraints should bind. Indeed, the upfront payment increases with the number of contracting periods, while the cost of lying stays constant $\left(\Delta \theta \underline{q}_{1}^{F B}\right)$.

Our results rely on the assumption that the cost parameter is perfectly correlated over time, and thus it considers an extreme ratchet effect. Indeed, if the regulator learns the firm's type in the first period, it is fully informed in the second period and captures all of the revenue. However, a more realistic assumption is to consider imperfectly correlated costs. Moreover, we could also assume as in Osmundsen (1998) and Martimort et al. (2018) that the cost parameter depends on the size of the stock. As a continuation to this work, it would be interesting to define the optimal renegotiation proof contracts. The renegotiation proof outcome usually lies between the outcome of full and non commitment. 


\section{References}

Bester, H. and Strausz, R. (2001). Contracting with imperfect commitment and the revelation principle: The single agent case. Econometrica, 69(4):1077-1098.

Boadway, R. and Keen, M. (2009). Theoretical Perspectives on Resource Tax Design. Working Papers 1206, Queen's University, Department of Economics.

Brito, D. L., Hamilton, J. H., Slutsky, S. M., and Stiglitz, J. E. (1991). Dynamic optimal income taxation with government commitment. Journal of Public Economics, 44(1):15 - 35 .

Collier, P. and Venables, A. J. (2010). International Rules for Trade in Natural Resources. Journal of Globalization and Development, 1(1):1-19.

Dillen, M. and Lundholm, M. (1996). Dynamic income taxation, redistribution, and the ratchet effect. Journal of Public Economics, 59(1):69 - 93.

Dionne, G. and Fluet, C. (2000). Full pooling in multi-period contracting with adverse selection and noncommitment. Review of Economic Design, 5(1):1-21.

Farhi, E., Sleet, C., Werning, I., and Yeltekin, S. (2012). Non-linear capital taxation without commitment. The Review of Economic Studies, 79(4):1469-1493.

Freixas, X., Guesnerie, R., and Tirole, J. (1985). Planning under incomplete information and the ratchet effect. The Review of Economic Studies, 52(2):173-191.

Gaudet, G. and Lassere, P. (2015). The management of natural resources under asymmetry of information. Annual Review of Resource Economics, 7(1):291-308.

Gaudet, G., Lassere, P., and Long, N. V. (1995). Optimal resource royalties with unknown and temporally independent extraction cost structures. International Economic Review, $36(3): 715-49$.

Humphreys, M., Sachs, J., and Stiglitz, J. E. (2007). Escaping the resource curse. Cambridge Univ Press. 
Hung, N. M., Poudou, J.-C., and Thomas, L. (2006). Optimal resource extraction contract with adverse selection. Resources Policy, 31(2):78-85.

Kolstad, I. and Wiig, A. (2009). It's the rents, stupid! the political economy of the resource curse. Energy Policy, 37(12):5317-5325.

Laffont, J.-J. and Tirole, J. (1988). The dynamics of incentive contracts. Econometrica, $56(5): 1153-1175$.

Laffont, J.-J. and Tirole, J. (1990). Adverse selection and renegotiation in procurement. The

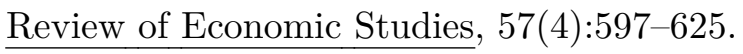

Martimort, D., Pouyet, J., and Ricci, F. (2018). Extracting information or resource? the hotelling rule revisited under asymmetric information. The RAND Journal of Economics, $49(2): 311-347$.

Osmundsen, P. (1998). Dynamic taxation of non-renewable natural resources under asymmetric information about reserves. Canadian Journal of Economics, 31(4):933-951.

Osmundsen, P. (2009). Time consistency in petroleum taxation:the case of norway. UiS Working Papers in Economics and Finance 2009/18, pages 25-27.

Robinson, J. A., Torvik, R., and Verdier, T. (2006). Political foundations of the resource curse. Journal of Development Economics, 79(2):447-468. Special Issue in honor of Pranab Bardhan.

Sachs, J. D. and Warner, A. M. (2001). The curse of natural resources. European Economic Review, 45(4-6):827-838. 


\section{Appendices}

To simplify the presentation of the proofs, we will use the following notations:

$$
\begin{aligned}
& \underline{Z} \equiv \underline{X}_{1}+\underline{X}_{2}-b S \\
& \bar{Z} \equiv \bar{X}_{1}+\bar{X}_{2}-b S \\
& \frac{\partial c(\theta, q)}{\partial q}=M C(\theta, q)=\theta+b q
\end{aligned}
$$

To compare the tax revenue, we will often use:

$$
\begin{aligned}
& u\left(\theta, q_{a}, \tau_{t}, p_{t}\right)-u\left(\theta, q_{b}, \tau_{t}, p_{t}\right)=\left(q_{a}-q_{b}\right)\left(X_{t}-\frac{b}{2}\left(q_{a}+q_{b}\right)\right) \\
& u\left(\theta, q_{1_{e}}^{F B}, 0, p_{1}\right)+\delta u\left(\theta, S-q_{1_{e}}^{F B}, 0, p_{2}\right)-u\left(\theta, \tilde{q}_{1}, 0, p_{1}\right)-\delta u\left(\theta, S-\tilde{q}_{1}, 0, p_{2}\right)=\frac{b}{2}(1+\delta)\left(q_{1_{e}}^{F B}-\tilde{q}_{1}\right)^{2}
\end{aligned}
$$

\section{A Benchmarks}

\section{Symmetric information}

Assuming that $S<S^{a}$, where $S^{a}$ is defined by (9), both types exhaust the stock. Note that $S<S^{a}$ if the price net of the marginal cost is sufficiently high as compared to the stock. If $S<S^{a}$ the extraction path satisfies:

$p_{1}-M C\left(\theta, q_{1}\right)=\delta\left(p_{2}-M C\left(\theta, S-q_{1}\right)\right)$

which defines $q_{1_{e}}^{F B}$ and $q_{2_{e}}^{F B}$ respectively given by (10) and (11). $\bar{q}_{1_{e}}^{F B}>0$ and $\underline{q}_{2_{e}}^{F B}>0$ if respectively:

$$
\begin{aligned}
& \bar{X}_{1}+\delta b S-\delta \bar{X}_{2}>0 \\
& \delta \underline{X}_{2}+b S-\underline{X}_{1}>0
\end{aligned}
$$


At each period, the quantity extracted is positive if the initial stock and the price at this period are relatively high, while the price at the other period is relatively low.

$\frac{\partial q_{1}^{F B}}{\partial \delta}=-\frac{X_{1}+X_{2}-b S}{b(1+\delta)^{2}}$ which is negative from $S<S^{a}$.

The tax revenue is:

$\tau_{1}^{F B}=\frac{X_{1}{ }^{2}}{2 b}-\frac{\delta^{2} Z^{2}}{2 b(1+\delta)^{2}} ; \quad \tau_{2}^{F B}=\frac{X_{2}^{2}}{2 b}-\frac{Z^{2}}{2 b(1+\delta)^{2}}$

Implementation. The regulator could force the firm to extract the socially optimal quantity $\left(q_{1_{e}}^{F B}\right.$ and $\left.q_{2_{e}}^{F B}\right)$ and ask for an upfront license fee $L^{F B}=\tau_{1}^{F B}+\delta \tau_{2}^{F B}$, or a license fee paid at each period with $L_{1}^{F B}=\tau_{1}^{F B}$ and $L_{2}^{F B}=\tau_{2}^{F B}$. The regulator could also impose the payment $L^{F B}$ (or $L_{1}^{F B}$ and $L_{2}^{F B}$ ), and delegate the extraction decisions to the firm. Indeed, the firm would only be able to pay the license fee if it actually extracts the optimal level since any other choice leaves it with a negative payoff.

Exclusion. In the paper, we focus on parameters that are such that an inefficient firm extracts a positive quantity at each period. Implicitly, we assume that the regulator has no incentives to exclude an inefficient firm. However, if the regulator excludes an inefficient firm, it receives: $V_{R}^{e x c}=\nu_{1}\left(\frac{\underline{X}_{1}{ }^{2}}{2 b}-\frac{\delta^{2} \underline{Z}^{2}}{2 b(1+\delta)^{2}}+\delta\left(\frac{\underline{X}_{2}{ }^{2}}{2 b}-\frac{\underline{Z}^{2}}{2 b(1+\delta)^{2}}\right)\right)$

\section{Full commitment}

(i) Production level:

If (18) is slack, at each period, an inefficient firm's production is given by:

$\left(1-\nu_{1}\right)\left(\bar{X}_{t}-b \bar{q}_{t}\right)-\nu_{1} \Delta \theta=0$, which defines $\bar{q}_{1}^{F C}$ and $\bar{q}_{2}^{F C}$ given by (20) and (21). The quantity extracted are positive if:

$\bar{X}_{1}\left(1-\nu_{1}\right)-\Delta \theta \nu_{1}>0$
$\bar{X}_{2}\left(1-\nu_{1}\right)-\Delta \theta \nu_{1}>0$ 
From $A 2_{a}$ and $A 2_{b}$, an inefficient firm produces a positive quantity if $\Delta \theta$ and $\nu_{1}$ are relatively low. If so, the trade off between rent extraction and efficiency favours efficiency. Indeed, if the probability to face an efficient firm and the size of the information asymmetry are relatively low, the informational rent is relatively low, and less likely given. As a result, the regulator is not willing to substantially distort the inefficient firm's extraction. $A 2_{a}$ and $A 2_{b}$ also imply that it is not optimal to exclude an inefficient firm. From $\bar{q}_{1}^{F C}$ and $\bar{q}_{2}^{F C}$, we obtain $S^{b}$ given by (22). $S^{b}<S^{a}$ implies that the stock may be exhausted by an inefficient under symmetric information, while it is not under asymmetric information with commitment. Indeed, the trade off between rent extraction and efficiency decreases the inefficient firm's extraction.

From $S>S^{b}, \frac{\delta\left(\bar{X}_{1}+\bar{X}_{2}-b S\right)}{b(1+\delta)}-\frac{\Delta \theta \nu_{1}}{b\left(1-\nu_{1}\right)}<0$, which implies from $(20)$, that $\bar{q}_{1}^{F C}<\bar{q}_{1_{e}}^{F B}$.

If $S<S^{b}$, the inefficient firm's extraction is given by:

$\left(1-\nu_{1}\right)\left(\bar{X}_{1}-b \bar{q}_{1}-\delta\left(\bar{X}_{2}-b\left(S-\bar{q}_{1}\right)\right)\right)-\nu_{1} \Delta \theta(1-\delta)=0$

The modified Hotelling's rule is given by:

$p_{1}-M C\left(\bar{\theta}, \bar{q}_{1}\right)-\frac{\Delta \theta \nu_{1}}{1-\nu_{1}}=\delta\left(p_{2}-M C\left(\bar{\theta}, S-\bar{q}_{1}\right)-\frac{\Delta \theta \nu_{1}}{1-\nu_{1}}\right) \quad\left(\overline{H R}^{F C}\right)$

which defines $\bar{q}_{1_{e}}^{F C}$ and $\bar{q}_{2_{e}}^{F C}$ respectively given by (23) and (24). $A 2_{c}$ ensures that $\bar{q}_{1_{e}}^{F C}>0$ :

$$
\left(\bar{X}_{1}-\delta \bar{X}_{2}+\delta b S\right)\left(1-\nu_{1}\right)-\Delta \theta \nu_{1}(1-\delta)>0
$$

Once again $A 2_{c}$ is satisfied if $\nu_{1}$ and $\Delta \theta$ are relatively low, and $A 2_{c}$ implies that it is not optimal to exclude an inefficient firm. $\frac{\partial \bar{q}_{1 e}^{F C}}{\partial \delta}=-\frac{\bar{Z}\left(1-\nu_{1}\right)-2 \nu_{1} \Delta \theta}{b(1+\delta)^{2}\left(1-\nu_{1}\right)}$ which is negative from $S<S^{b}$.

Regardless of (18) binding, (16) is always satisfied.

(16) is satisfied if $\underline{q}_{1}-\bar{q}_{1}+\delta\left(\underline{q}_{2}-\bar{q}_{2}\right) \geq 0$ 
If $S>S^{b}$, the production is $\underline{q}_{1_{e}}^{F B}, \underline{q}_{2_{e}}^{F B}, \bar{q}_{1}^{F C}$ and $\bar{q}_{2}^{F C}$

$\underline{q}_{1_{e}}^{F B}-\bar{q}_{1}^{F C}+\delta\left(\underline{q}_{2_{e}}^{F B}-\bar{q}_{2}^{F C}\right)=\frac{(1-\delta)^{2} \Delta \theta}{b\left(1-\nu_{1}\right)(1+\delta)}-\frac{2 \delta\left(\bar{Z}\left(1-\nu_{1}\right)-2 \nu_{1} \Delta \theta\right)}{b(1+\delta)\left(1-\nu_{1}\right)}$

$S>S^{b}$ implies $\bar{Z}\left(1-\nu_{1}\right)-2 \nu_{1} \Delta \theta<0$, hence, (31) is positive and (16) is satisfied.

If $S<S^{b}$, the production is $\underline{q}_{1_{e}}^{F B}, \underline{q}_{2_{e}}^{F B}=S-\underline{q}_{2_{e}}^{F B}, \bar{q}_{1_{e}}^{F C}$ and $\bar{q}_{2_{e}}^{F C}=S-\bar{q}_{2_{e}}^{F C}$

$\underline{q}_{1_{e}}^{F B}-\bar{q}_{1_{e}}^{F C}+\delta\left(S-\underline{q}_{1_{e}}^{F B}-S+\bar{q}_{1_{e}}^{F C}\right)=\left(\underline{q}_{1_{e}}^{F B}-\bar{q}_{1_{e}}^{F C}\right)(1-\delta)=\frac{(1-\delta)^{2} \Delta \theta^{2}}{b\left(1-\nu_{1}\right)(1+\delta)}$

(32) is positive and (16) is satisfied.

(ii) Tax revenue:

We denote by $\underline{\tau}_{1}^{F C^{*}}, \underline{\tau}_{2}^{F C^{*}}, \bar{\tau}_{1}^{F C^{*}}$ and $\bar{\tau}_{2}^{F C^{*}}$ the equilibrium tax revenue when $S>S^{b}$, while $\underline{\tau}_{1}^{F C^{* *}}, \underline{\tau}_{2}^{F C^{* *}}, \bar{\tau}_{1}^{F C^{* *}}$ and $\bar{\tau}_{2}^{F C^{* *}}$ denote the equilibrium tax revenue when $S<S^{b}$.

If $S>S^{b}$

$\underline{\tau}_{1}^{F C^{*}}+\delta \underline{\tau}_{2}^{F C^{*}}=\underline{\tau}_{1}^{F B}+\delta \underline{\tau}_{2}^{F B}-\Delta \theta\left(\bar{q}_{1}^{F C}+\delta \bar{q}_{2}^{F C}\right)=\underline{\tau}_{1}^{F B}+\delta \underline{\tau}_{2}^{F B}-\Delta \theta\left(\frac{\bar{X}_{1}}{b}+\frac{\delta \bar{X}_{2}}{b}-\frac{(1+\delta) \Delta \theta \nu_{1}}{b\left(1-\nu_{1}\right)}\right)$

From $(29), \bar{\tau}_{t}^{F C^{*}}=\bar{\tau}_{t}^{F B}-\left(\bar{q}_{t_{e}}^{F B}-\bar{q}_{t}^{F C}\right)\left(\bar{X}_{t}-\frac{b}{2}\left(\bar{q}_{t_{e}}^{F B}+\bar{q}_{t}^{F C}\right)\right)$, and thus:

$\bar{\tau}_{1}^{F C^{*}}=\bar{\tau}_{1}^{F B}-\left(\frac{\Delta \theta \nu_{1}}{b\left(1-\nu_{1}\right)}-\frac{\delta \bar{Z}}{b(1+\delta)}\right) \frac{b}{2}\left(\frac{\Delta \theta \nu_{1}}{b\left(1-\nu_{1}\right)}+\frac{\delta \bar{Z}}{b(1+\delta)}\right)=\bar{\tau}_{1}^{F B}-\frac{\Delta \theta^{2} \nu_{1}^{2}}{2 b\left(1-\nu_{1}\right)^{2}}+\frac{\delta^{2} \bar{Z}^{2}}{2 b(1+\delta)^{2}}$

$\bar{\tau}_{2}^{F C^{*}}=\bar{\tau}_{2}^{F B}-\left(\frac{\Delta \theta \nu_{1}}{b\left(1-\nu_{1}\right)}-\frac{\bar{Z}}{b(1+\delta)}\right) \frac{b}{2}\left(\frac{\Delta \theta \nu_{1}}{b\left(1-\nu_{1}\right)}+\frac{\bar{Z}}{b(1+\delta)}\right)=\bar{\tau}_{2}^{F B}-\frac{\Delta \theta^{2} \nu_{1}^{2}}{2 b\left(1-\nu_{1}\right)^{2}}+\frac{\bar{Z}^{2}}{2 b(1+\delta)^{2}}$

From, $\bar{\tau}_{1}^{F C^{*}}+\delta \bar{\tau}_{2}^{F C^{*}}=\bar{\tau}_{1}^{F B}+\delta \bar{\tau}_{2}^{F B}-\frac{\Delta \theta^{2} \nu_{1}^{2}(1+\delta)}{2 b\left(1-\nu_{1}\right)^{2}}+\frac{\delta \bar{Z}^{2}}{2 b(1+\delta)}$, the tax loss $T S$ is given by:

$$
\begin{aligned}
T S & =\nu_{1} \Delta \theta\left(\frac{\bar{X}_{1}}{b}+\frac{\delta \bar{X}_{2}}{b}-\frac{(1+\delta) \Delta \theta \nu_{1}}{b\left(1-\nu_{1}\right)}\right)+\frac{\Delta \theta^{2} \nu_{1}^{2}(1+\delta)}{2 b\left(1-\nu_{1}\right)}-\frac{\delta \bar{Z}^{2}\left(1-\nu_{1}\right)}{2 b(1+\delta)} \\
& =\Delta \theta \nu_{1}\left(\frac{\delta \bar{X}_{2}}{b}+\frac{\bar{X}_{1}}{b}\right)-\frac{(1+\delta) \Delta \theta^{2} \nu_{1}^{2}}{2 b\left(1-\nu_{1}\right)}-\frac{\delta \bar{Z}^{2}\left(1-\nu_{1}\right)}{2 b(1+\delta)}
\end{aligned}
$$


Implementation. The regulator can propose a menu of two contracts. A first contract composed by a royalty paid on the revenue $\bar{r}_{t}^{F C}=\frac{\Delta \theta \nu_{1}}{p_{t}\left(1-\nu_{1}\right)}$ and a license fee $\bar{L}_{t}^{F C^{*}}=\frac{\left(\bar{X}_{t}\left(1-\nu_{1}\right)-\nu_{1} \Delta \theta\right)^{2}}{2 b\left(1-\nu_{1}\right)^{2}}$ (or a fee $\bar{L}^{F C^{*}}=\bar{L}_{1}^{F C^{*}}+\delta \bar{L}_{2}^{F C^{*}}$ given upfront). This contract specifies at each period an upper limit to the extraction $q_{t} \leq \bar{q}_{t}^{F C}$. The second contract should only impose a license fee at each period $\underline{L}_{t}^{F C^{*}}=\underline{\tau}_{t}^{F B}-\Delta \theta \bar{q}_{t}^{F C}$ (or an upfront license fee $\underline{L}^{F C^{*}}=\underline{\tau}_{1}^{F C^{*}}+\delta \underline{\tau}_{2}^{F C^{*}}$ ).

If $S<S^{b}$

$$
\begin{aligned}
\underline{\tau}_{1}^{F C^{* *}}+\delta \underline{\tau}_{2}^{F C^{* *}} & =\underline{\tau}_{1}^{F B}+\delta \underline{\tau}_{2}^{F B}-\Delta \theta\left(\bar{q}_{1_{e}}^{F C}+\delta \bar{q}_{2_{e} C}^{F C}\right) \\
& =\underline{\tau}_{1}^{F B}+\delta \underline{\tau}_{2}^{F B}-\Delta \theta\left(\frac{\bar{X}_{1}}{b}+\frac{\delta \bar{X}_{2}}{b}-\frac{2 \delta \bar{Z}}{b(1+\delta)}-\frac{(1-\delta)^{2} \Delta \theta \nu_{1}}{b\left(1-\nu_{1}\right)(1+\delta)}\right)
\end{aligned}
$$

Using (30), we obtain the tax revenue for an inefficient firm:

$\bar{\tau}_{1}^{F C^{* *}}+\delta \bar{\tau}_{2}^{F C^{* *}}=\bar{\tau}_{1}^{F B}+\delta \bar{\tau}_{2}^{F B}-\frac{b}{2}(1+\delta)\left(\frac{(1-\delta) \nu_{1} \Delta \theta}{b(1+\delta)\left(1-\nu_{1}\right)}\right)^{2}=\bar{\tau}_{1}^{F B}+\delta \bar{\tau}_{2}^{F B}-\frac{(1-\delta)^{2} \nu_{1}^{2} \Delta \theta^{2}}{2 b(1+\delta)\left(1-\nu_{1}\right)^{2}}$

The tax loss is given by:

$$
\begin{aligned}
T S & =\nu_{1} \Delta \theta\left(\frac{\bar{X}_{1}}{b}+\frac{\delta \bar{X}_{2}}{b}-\frac{2 \delta \bar{Z}}{b(1+\delta)}-\frac{(1-\delta)^{2} \Delta \theta \nu_{1}}{b\left(1-\nu_{1}\right)(1+\delta)}\right)+\frac{(1-\delta)^{2} \nu_{1}^{2} \Delta \theta^{2}}{2 b(1+\delta)\left(1-\nu_{1}\right)} \\
& =\nu_{1} \Delta \theta\left(\frac{\bar{X}_{1}}{b}+\frac{\delta \bar{X}_{2}}{b}-\frac{2 \delta \bar{Z}}{b(1+\delta)}\right)-\frac{(1-\delta)^{2} \nu_{1}^{2} \Delta \theta^{2}}{2 b(1+\delta)\left(1-\nu_{1}\right)}
\end{aligned}
$$

Implementation. The regulator can propose a menu of two contracts. A first contract composed by the same royalty as before $\left(\bar{r}_{t}^{F C}\right)$ and the license fees $\bar{L}_{1}^{F C^{* *}}=\bar{\tau}_{1}^{F B}+\frac{\Delta \theta^{2}(1-\delta)(1+3 \delta) \nu_{1}{ }^{2}}{2 b(\delta+1)^{2}\left(1-\nu_{1}\right)^{2}}+$ $\frac{2 \Delta \theta \delta^{2} \nu_{1} \bar{Z}}{b(1+\delta)^{2}\left(1-\nu_{1}\right)}-\frac{\Delta \theta \bar{X}_{1} \nu_{1}}{b\left(1-\nu_{1}\right)}$ and $\bar{L}_{2}^{F C^{* *}}=\bar{\tau}_{2}^{F B}-\frac{\Delta \theta^{2} \nu_{1}^{2}(1-\delta)(3+\delta)}{2 b(1+\delta)^{2}\left(1-\nu_{1}\right)^{2}}+\frac{2 \Delta \theta \nu_{1} \bar{Z}}{b(1+\delta)^{2}\left(1-\nu_{1}\right)}-\frac{\Delta \theta \bar{X}_{2} \nu_{1}}{b\left(1-\nu_{1}\right)}$ (or a fee $\bar{L}^{F C^{* *}}=\bar{L}_{1}^{F C^{* *}}+\delta \bar{L}_{2}^{F C^{* *}}$ given upfront). This contract specifies an upper limit to the first-period extraction $q_{1} \leq \bar{q}_{1_{e}}^{F C}$. The second contract should only impose a license fee at each period $\underline{L}_{t}^{F C^{* *}}=\underline{\tau}_{t}^{F B}-\Delta \theta \bar{q}_{t_{e}}^{F C}$ (or an upfront license fee $\underline{L}^{F C^{* *}}=\underline{\tau}_{1}^{F C^{* *}}+\delta \underline{\tau}_{2}^{F C^{* *}}$ ). 


\section{B Second-period contracts without commitment}

For a given stock $S_{2}=S-q_{1}$ and updated belief $\nu_{2}$, the regulator solves:

$\max _{\left\{\underline{q}_{2}, \underline{\tau}_{2}, \bar{q}_{2}, \bar{\tau}_{2}\right\}} V_{R_{2}}^{N C}=\nu_{2} \underline{\tau}_{2}+\left(1-\nu_{2}\right) \bar{\tau}_{2}$ subject to

$u\left(\underline{\theta}_{2} \underline{q}_{2}, \underline{\tau}_{2}, p_{2}\right) \geq 0$

$u\left(\bar{\theta}, \bar{q}_{2}, \bar{\tau}_{2}, p_{2}\right) \geq 0$

$u\left(\underline{\theta}, \underline{q}_{2}, \underline{\tau}_{2}, p_{2}\right) \geq u\left(\underline{\theta}, \bar{q}_{2}, \bar{\tau}_{2}, p_{2}\right)$

$u\left(\bar{\theta}, \bar{q}_{2}, \bar{\tau}_{2}, p_{2}\right) \geq u\left(\bar{\theta}, \underline{q}_{2}, \underline{\tau}_{2}, p_{2}\right)$

$\underline{q}_{2} \leq S_{2}$

$\bar{q}_{2} \leq S_{2}$

At the optimum, (34) and (35) bind. From them, $\bar{\tau}_{2}^{N C}=\bar{\tau}_{2}^{R}, \underline{\tau}_{2}^{N C}=\underline{\tau}_{2}^{R}-\Delta \theta \bar{q}_{2}$. (34) $\Leftrightarrow$ $\Delta \theta \bar{q}_{2}\left(\nu_{2}\right) \geq 0$, which is always satisfied. (36) $\Leftrightarrow \Delta \theta\left(\underline{q}_{2}-\bar{q}_{2}\right) \geq 0$, which is satisfied if $\underline{q}_{2} \geq \bar{q}_{2}$. By inserting $\bar{\tau}_{2}^{N C}=\bar{\tau}_{2}^{R}$ and $\underline{\tau}_{2}^{N C}=\underline{\tau}_{2}^{R}-\Delta \theta \bar{q}_{2}$ into $V_{R_{2}}^{N C}$, the problem becomes:

$\max _{\left\{\underline{q}_{2}, \bar{q}_{2}\right\}} V_{R_{2}}^{N C}=\nu_{2}\left(\underline{\tau}_{2}^{R}-\Delta \theta \bar{q}_{2}\right)+\left(1-\nu_{2}\right) \bar{\tau}_{2}^{R}$ subject to $(36),(37),(38)$

Solving the problem gives:

$\underline{q}_{2}^{N C}=\underline{q}_{2}^{F B}$ if $S_{2}>\frac{\underline{X}_{2}}{b}$, if not $\underline{q}_{2}=S_{2} \cdot \bar{q}_{2}^{N C}=\bar{q}_{2}^{F B}-\frac{\Delta \theta \nu_{2}}{b\left(1-\nu_{2}\right)}$ if $S_{2}>\frac{\bar{X}_{2}}{b}-\frac{\Delta \theta \nu_{2}}{b\left(1-\nu_{2}\right)}$, if not, $\bar{q}_{2}=S_{2}$. From $\frac{X_{2}}{b}>\frac{\bar{X}_{2}}{b}-\frac{\Delta \theta \nu_{2}}{b\left(1-\nu_{2}\right)},(37)$ may bind, while (38) is slack.

If neither (37) nor (38) bind, $\underline{q}_{2}-\bar{q}_{2}=\frac{X_{2}}{b}-\frac{\bar{X}_{2}}{b}+\frac{\Delta \theta \nu_{2}}{b\left(1-\nu_{2}\right)}=\frac{\Delta \theta}{b\left(1-\nu_{2}\right)}$, thus (36) is satisfied.

If both (37) and (38) bind, $\underline{q}_{2}-\bar{q}_{2}=S_{2}-S_{2}=0$, thus (36) is satisfied.

If only (37) binds, $\underline{q}_{2}-\bar{q}_{2}=S_{2}-\frac{\bar{X}_{2}}{b}+\frac{\Delta \theta \nu_{2}}{b\left(1-\nu_{2}\right)}$ which is positive from (38) slack, thus (36) is satisfied.

$\bar{\tau}_{2 / \bar{C}_{1}}^{R}$ and $\underline{\tau}_{2 / \bar{C}_{1}}^{R}$ (respectively $\bar{\tau}_{2 / \underline{C}_{1}}^{R}$ and $\underline{\tau}_{2 / \underline{C}_{1}}^{R}$ ) denote the taxes that are such that the regulator captures the entire net revenue knowing that $\bar{C}_{1}$ (respectively $\underline{C}_{1}$ ) was chosen during the first period. 


\section{Proof of Proposition 1}

\section{C.1 Production}

If only ( $\underline{\text { IC }) ~ b i n d s, ~} y=0, \underline{\nu}_{2}=1, \bar{q}_{2 / \underline{C}_{1}}=0$, and $\bar{q}_{2 / \bar{C}_{1}}=\bar{q}_{2}^{F B}-\frac{\Delta \theta(1-x)}{b\left(1-\nu_{1}\right)}$ if the stock is not exhausted, or $\bar{q}_{2 / \bar{C}_{1}}=S-\bar{q}_{1}$ otherwise.

From $(\overline{P C})$ binding: $\bar{\tau}_{1}^{N C}=\bar{\tau}_{1}^{R}$, from $\bar{q}_{2 / \underline{C}_{1}}=0$, ( $\left.\underline{\mathrm{IC}}\right)$ binding implies:

$u\left(\underline{\theta}, \underline{q}_{1}, \underline{\tau}_{1}, p_{1}\right)=u\left(\underline{\theta}, \bar{q}_{1}, \bar{\tau}_{1}^{R}, p_{1}\right)+\delta \Delta \theta \bar{q}_{2 / \bar{C}_{1}}$. Using $\bar{\tau}_{1}^{N C}=\bar{\tau}_{1}^{R},(\underline{\text { IC }})$ becomes:

$p_{1} \underline{q}_{1}-c\left(\underline{\theta}, \underline{q}_{1}\right)-\underline{\tau}_{1}^{N C}=c\left(\bar{\theta}, \bar{q}_{1}\right)-c\left(\underline{\theta}, \bar{q}_{1}\right)+\delta \Delta \theta \bar{q}_{2 / \bar{C}_{1}}$, which gives:

$\underline{\tau}_{1}^{N C}=\underline{\tau}_{1}^{R}-\Delta \theta\left(\bar{q}_{1}+\delta \bar{q}_{2 / \bar{C}_{1}}\right)$

The regulator's payoff (40) is obtained by replacing $y=0, \bar{\tau}_{1}^{N C}=\bar{\tau}_{1}^{R}$, and the tax given by (39) into (25):

$$
\begin{aligned}
\max _{\left\{\underline{q}_{1}, \bar{q}_{1}, x\right\}} V_{R}^{N C} & =\nu_{1} x\left(\underline{\tau}_{1}^{R}-\Delta \theta\left(\bar{q}_{1}+\delta \bar{q}_{2 / \bar{C}_{1}}\right)+\delta \underline{\tau}_{2 / \underline{C}_{1}}^{R}\right)+\left(1-\nu_{1} x\right) \bar{\tau}_{1}^{R} \\
& +\delta \nu_{1}(1-x)\left(\underline{\tau}_{2 / \bar{C}_{1}}^{R}-\Delta \theta \bar{q}_{2 / \bar{C}_{1}}\right)+\delta\left(1-\nu_{1}\right) \bar{\tau}_{2 / \bar{C}_{1}}^{R} \\
& \text { subject to }\left(S C_{1}\right),\left(S C_{2}\right),\left(S C_{3}\right),(\overline{I C})
\end{aligned}
$$

The first part of (40) is the expected tax revenue if the firm is efficient and reveals its type. If so, in the first period, the regulator compensates an efficient firm for revealing its type in the first period, and for the forgone informational rent. In the second period, the regulator captures the entire revenue. The second part is the expected tax revenue if the firm is efficient and lies or if it is inefficient. The third part is the second-period expected tax revenue if the firm is efficient and lied in the first period. If so, the firm gets an informational rent. Finally, the last part is the second-period expected tax revenue if the firm is inefficient.

Assuming that $S<S^{a}$, an efficient firm's production is given by:

$\frac{\partial V_{R}^{N C}}{\partial \underline{q}_{1}}=\nu_{1} x\left(\frac{\partial \underline{\tau}_{1}^{R}}{\partial \underline{q}_{1}}+\delta \frac{\partial \underline{\tau}_{2 / \underline{C}_{1}}^{R}}{\partial \underline{q}_{1}}\right)=0 \Leftrightarrow \underline{q}_{1}=\underline{q}_{1_{e}}^{F B}$ 
- If $\left(S C_{1}\right)$ and $\left(S C_{3}\right)$ bind

$$
\begin{aligned}
\frac{\partial V_{R}^{N C}}{\partial \bar{q}_{1}} & =-\Delta \theta \nu_{1} x+\left(1-\nu_{1} x\right) \frac{\partial \bar{\tau}_{1}^{R}}{\partial \bar{q}_{1}}+\delta \nu_{1}(1-x) \frac{\partial \underline{\tau}_{2 / \bar{C}_{1}}^{R}}{\partial \bar{q}_{1}} \\
& =-\Delta \theta \nu_{1} x+\left(1-\nu_{1} x\right)\left(\bar{X}_{1}-b \bar{q}_{1}\right)-\delta \nu_{1}(1-x)\left(\underline{X}_{2}-b\left(S-\bar{q}_{1}\right)\right)=0
\end{aligned}
$$

From (42), an inefficient produces:

$\hat{\bar{q}}_{1}=\bar{q}_{1}^{F C}-\frac{\left(\delta\left(\underline{X}_{1}+\underline{X}_{2}-b S\right)\left(1-\nu_{1}\right)-(1+\delta) \Delta \theta\right) \nu_{1}(1-x)}{b\left(1-\nu_{1}\right)\left(1-\nu_{1} x+\delta \nu_{1}(1-x)\right)}$

To determine the optimal level of separation, we replace $\hat{\bar{q}}_{1}$ and $\underline{q}_{1 e}^{F B}$ into (40) and we differentiate and solve with respect to $x$. From the envelope theorem, the optimal separation level $x^{*}$ has no effect on the inefficient firm's first-period production $\hat{\bar{q}}_{1}$.

$$
\begin{aligned}
\frac{\partial V_{R}^{N C}}{\partial x} & =\nu_{1}\left(\underline{\tau}_{1}^{R}-\bar{\tau}_{1}^{R}-\Delta \theta \bar{q}_{1}+\delta \underline{\tau}_{2 / \underline{C}_{1}}^{R}-\delta \underline{\tau}_{2 / \bar{C}_{1}}^{R}\right)+\delta\left(\left(1-\nu_{1}\right) \frac{\partial \bar{\tau}_{2 / \bar{C}_{1}}^{R}}{\partial \bar{q}_{2}(x)}+\nu_{1} \Delta \theta\right) \frac{\partial \bar{q}_{2 / \bar{C}_{1}}}{\partial x} \\
& =\frac{\left((1+\delta) \Delta \theta-\delta \underline{Z}\left(1-\nu_{1}\right)\right)^{2} \nu_{1}}{2 b(1+\delta)\left(1-\nu_{1} x+\delta \nu_{1}(1-x)\right)^{2}}-\frac{\delta \Delta \theta^{2} \nu_{1}^{2} x}{b\left(1-\nu_{1}\right)}
\end{aligned}
$$

Computed at $x=1, \frac{\partial V_{R}^{N C}}{\partial x}=\frac{\left((1+\delta) \Delta \theta-\delta \underline{Z}\left(1-\nu_{1}\right)\right)^{2} \nu_{1}}{2 b(1+\delta)\left(1-\nu_{1}\right)^{2}}-\frac{\delta \Delta \theta^{2} \nu_{1}^{2}}{b\left(1-\nu_{1}\right)}>0$

Full-separating contracts are proposed $\left(x^{*}=1\right)$, an inefficient firm produces $\bar{q}_{1}^{F C}$ and $\bar{q}_{2}^{F B}$. These contracts are implemented if $S>S^{c}$ where $S^{c}$ is defined in (26), and if $A 3_{a}$ is satisfied. The latter being satisfied if the following equation is positive:

$$
\begin{aligned}
& (1+\delta)(\bar{\theta}-\underline{\theta})-\delta\left(1-\nu_{1}\right)\left((1+\delta)\left(p_{2}-\bar{\theta}\right)+p_{1}+p_{2}-2 \underline{\theta}-b S\right) \\
& \frac{\partial(43)}{\partial p_{2}}=-\delta(\delta+2)\left(1-\nu_{1}\right)<0, \frac{\partial(43)}{\partial p_{1}}=-\delta\left(1-\nu_{1}\right)<0, \frac{\partial(43)}{\partial \bar{\theta}}=(1+\delta)\left(1+\delta\left(1-\nu_{1}\right)\right)>0 \\
& \frac{\partial(43)}{\partial \underline{\theta}}=-\left(2 \delta \nu_{1}+1-\delta\right)<0, \frac{\partial(43)}{\partial S}=-b \delta\left(1-\nu_{1}\right)<0 \\
& \frac{\partial(43)}{\partial \nu_{1}}=\delta(1+\delta)\left(p_{2}-\bar{\theta}\right)+\delta\left(p_{1}+p_{2}-\underline{\theta}-b S\right) \\
& \frac{\partial(43)}{\partial \nu_{1}}>0 \text { since by assumption } p_{2}-\bar{\theta}>0 \text { and } S<S^{a} \text { implies } p_{1}+p_{2}-2 \underline{\theta}-b S>0 .
\end{aligned}
$$


- If all $S C$ bind

In the second period, if $\bar{C}_{1}$ is chosen, both firms produce $S-\bar{q}_{1}$

$$
\begin{aligned}
\frac{\partial V_{R}^{N C}}{\partial \bar{q}_{1}}= & -\nu_{1} x \Delta \theta(1-\delta)+\left(1-\nu_{1} x\right) \frac{\partial \bar{\tau}_{1}^{R}}{\partial \bar{q}_{1}}+\delta \nu_{1}(1-x)\left(\frac{\partial \underline{\tau}_{2 / \bar{C}_{1}}^{R}}{\partial \bar{q}_{1}}+\Delta \theta\right)+\delta\left(1-\nu_{1}\right) \frac{\partial \bar{\tau}_{2 / \bar{C}_{1}}^{R}}{\partial \bar{q}_{1}} \\
= & -\nu_{1} x \Delta \theta(1-\delta)+\left(1-\nu_{1} x\right)\left(\bar{X}_{1}-b \bar{q}_{1}\right)-\delta \nu_{1}(1-x)\left(\underline{X}_{2}-b\left(S-\bar{q}_{1}\right)-\Delta \theta\right) \\
& -\delta\left(1-\nu_{1}\right)\left(\bar{X}_{2}-b\left(S-\bar{q}_{1}\right)\right) \\
= & -\nu_{1} x \Delta \theta(1-\delta)+\left(1-\nu_{1} x\right)\left(\bar{X}_{1}-b \bar{q}_{1}-\delta\left(\bar{X}_{2}-b\left(S-\bar{q}_{1}\right)\right)\right)=0
\end{aligned}
$$

From (44), an inefficient produces:

$$
\hat{\bar{q}}_{1 e}=\bar{q}_{1_{e}}^{F B}-\frac{\Delta \theta \nu_{1} x(1-\delta)}{b(1+\delta)\left(1-\nu_{1} x\right)}=\bar{q}_{1_{e}}^{F C}+\frac{(1-\delta)(1-x) \Delta \theta \nu_{1}}{b(1+\delta)\left(1-\nu_{1}\right)\left(1-\nu_{1} x\right)}
$$

We obtain the optimal separation level by replacing $\hat{\bar{q}}_{1_{e}}$ and $\underline{q}_{1 e}^{F B}$ into (40), and by differentiating and solve with respect to $x$.

$\frac{\partial V_{R}^{N C}}{\partial x}=\nu_{1}\left(\underline{\tau}_{1}^{R}-\bar{\tau}_{1}^{R}-\Delta \theta \bar{q}_{1}+\delta \underline{\tau}_{2 / \underline{C}_{1}}^{R}-\delta \underline{\tau}_{2 / \bar{C}_{1}}^{R}\right)$

Using $\tau_{t}^{R}=X_{t} q_{t}-\frac{b}{2} q_{t}^{2}$, (46) can be rewritten as:

$$
\begin{aligned}
\frac{\partial V_{R}^{N C}}{\partial x} & =\nu_{1}\left(\underline{X}_{1} \underline{q}_{1_{e}}^{F B}-\bar{X}_{1} \hat{\bar{q}}_{1_{e}}-\Delta \theta \hat{\bar{q}}_{1_{e}}+\delta \underline{X}_{2}\left(S-\underline{q}_{1_{e}}^{F B}-\left(S-\hat{\bar{q}}_{1_{e}}\right)\right)\right) \\
& +\nu_{1} \frac{b}{2}\left(\hat{\bar{q}}_{1_{e}}^{2}-\underline{q}_{1_{e}}^{F B^{2}}+\delta\left(S-\hat{\bar{q}}_{1_{e}}\right)^{2}-\delta\left(S-\underline{q}_{1_{e}}^{F B}\right)^{2}\right)
\end{aligned}
$$

Using

$$
\begin{aligned}
& \underline{X}_{1} \underline{q}_{1_{e}}^{F B}-\bar{X}_{1} \hat{\bar{q}}_{1_{e}}-\Delta \theta \hat{\bar{q}}_{1_{e}}=\underline{X}_{1}\left(\underline{q}_{1_{e}}^{F B}-\hat{\bar{q}}_{1_{e}}\right) \\
& \hat{\bar{q}}_{1_{e}}^{2}-\underline{q}_{1_{e}}^{F B^{2}}+\delta\left(S-\hat{\bar{q}}_{1_{e}}\right)^{2}-\delta\left(S-\underline{q}_{1_{e}}^{F B}\right)^{2}=\left(\underline{q}_{1_{e}}^{F B}-\hat{\bar{q}}_{1_{e}}\right)\left(2 \delta S-(1+\delta)\left(\underline{q}_{1_{e}}^{F B}+\hat{\bar{q}}_{1_{e}}\right)\right)
\end{aligned}
$$


(46) becomes:

$\nu_{1}\left(\underline{q}_{1_{e}}^{F B}-\hat{\bar{q}}_{1_{e}}\right)(\underbrace{X_{1}-\delta \underline{X}_{2}+\delta b S}_{=b(1+\delta) \underline{q}_{1_{e}}^{F B}}-(1+\delta) \frac{b}{2}\left(\underline{q}_{1_{e}}^{F B}+\hat{\bar{q}}_{1}\right))=\frac{b \nu_{1}(1+\delta)}{2}\left(\underline{q}_{1_{e}}^{F B}-\hat{\bar{q}}_{1_{e}}\right)^{2}>0$

Hence, $x^{*}=1$, and from (45), an inefficient firm produces $q_{1_{e}}^{F C}$. These contracts are implemented if $S<S^{c}$ where $S^{c}$ is defined in (26). $S^{c}>S^{b}$, indeed full separation implies that the inefficient firm's second-period production is not distorted by a second-period informational rent, while it is distorted with commitment. Moreover, these contracts are implemented if $A 3_{b}$ is satisfied, that is, if the following equation is positive:

$(1-\delta)\left(1-\delta \nu_{1}\right)(\bar{\theta}-\underline{\theta})-\delta\left(1-\nu_{1}\right)\left(\delta\left(p_{2}-\bar{\theta}\right)-\left(p_{1}-\bar{\theta}\right)+b S\right)$

$\frac{\partial(47)}{\partial p_{2}}=-\delta^{2}\left(1-\nu_{1}\right)<0, \frac{\partial(47)}{\partial p_{1}}=\delta\left(1-\nu_{1}\right)>0, \frac{\partial(47)}{\partial \bar{\theta}}=(1-\delta)^{2}>0$,

$\frac{\partial(47)}{\partial \underline{\theta}}=-(1-\delta)\left(1-\delta \nu_{1}\right)<0, \frac{\partial(47)}{\partial S}=-b \delta\left(1-\nu_{1}\right)<0, \frac{\partial(47)}{\partial \nu_{1}}=\delta\left(\delta\left(p_{2}-\underline{\theta}\right)-\left(p_{1}-\underline{\theta}\right)+b S\right)$.

$\bar{q}_{2_{e}}^{F C}>0$ implies $\frac{\partial(47)}{\partial \nu_{1}}>0$ since $\bar{q}_{2_{e}}^{F C}=\frac{\delta\left(p_{2}-\underline{\theta}\right)-\left(p_{1}-\underline{\theta}\right)+b S}{b(1+\delta)}$

\section{C.2 Tax revenue}

We denote by $\underline{\tau}_{1}^{N C *}, \underline{\tau}_{2}^{N C *}, \bar{\tau}_{1}^{N C *}$ and $\bar{\tau}_{2}^{N C *}$ the equilibrium tax revenue when $S>S^{c}$, while $\underline{\tau}_{1}^{N C * *}, \underline{\tau}_{2}^{N C * *}, \bar{\tau}_{1}^{N C * *}$ and $\bar{\tau}_{2}^{N C * *}$ denote the equilibrium tax revenue when $S<S^{c}$.

- If $S^{c}<S<S^{a}$

$$
\begin{aligned}
\underline{\tau}_{1}^{N C *}+\delta \underline{\tau}_{2}^{N C *} & =\underline{\tau}_{1}^{F C *}+\delta \underline{\tau}_{2}^{F C *}-\Delta \theta \delta\left(\bar{q}_{2}^{F B}-\bar{q}_{2}^{F C}\right)=\underline{\tau}_{1}^{F C *}+\delta \underline{\tau}_{2}^{F C *}-\frac{\delta \nu_{1} \Delta \theta^{2}}{b\left(1-\nu_{1}\right)} \\
\bar{\tau}_{1}^{N C *}+\delta \bar{\tau}_{2}^{N C *} & =\bar{\tau}_{1}^{F C *}+\delta \bar{\tau}_{2}^{F C *}-\delta\left(\bar{q}_{2}^{F C}-\bar{q}_{2}^{F B}\right)\left(\bar{X}_{2}-\frac{b}{2}\left(\bar{q}_{2}^{F C}+\bar{q}_{2}^{F B}\right)\right) \\
& =\bar{\tau}_{1}^{F C *}+\delta \bar{\tau}_{2}^{F C *}+\frac{\delta \nu_{1}^{2} \Delta \theta^{2}}{2 b\left(1-\nu_{1}\right)^{2}}
\end{aligned}
$$

As compared to full commitment, the tax revenue is lower, the tax cut is given by $\frac{\delta \nu_{1}^{2} \Delta \theta^{2}}{2 b\left(1-\nu_{1}\right)}$. 
Implementation. During the first period, the regulator can propose a menu of two contracts. A first contract composed by the same royalty as before $\left(\bar{r}_{1}^{F C}\right)$, a license fee $\bar{L}_{1}^{N C^{*}}=\bar{L}_{1}^{F C^{*}}$, and an upper limit to the extraction $q_{1} \leq \bar{q}_{1}^{F C}$. A second contract only imposing a license fee $\underline{L}_{1}^{N C^{*}}=\underline{\tau}_{1}^{F B}-\Delta \theta\left(\frac{\bar{X}_{1}}{b}+\frac{\delta \bar{X}_{2}}{b}-\frac{\Delta \theta \nu_{1}}{b\left(1-\nu_{1}\right)}\right)$, and a minimum quantity to extract $q_{1} \geq \underline{q}_{1_{e}}^{F B}$. If during the first period, the first contract was chosen, the second-period contract only specifies a license fee $\bar{L}_{2}^{N C^{*}}=\frac{\bar{X}_{2}{ }^{2}}{2 b}$ and restricts the extraction to $q_{2} \leq \bar{q}_{2}^{F B}$. If during the first period, the second contract was chosen, the second-period contract only specifies a license fee $\underline{L}_{2}^{N C^{*}}=\underline{\tau}_{2}^{F B}$.

- If $S^{b}<S<S^{c}$

$$
\begin{aligned}
\underline{\tau}_{1}^{N C * *}+\delta \underline{\tau}_{2}^{N C * *} & =\underline{\tau}_{1}^{F C *}+\delta \underline{\tau}_{2}^{F C *}-\Delta \theta\left(\bar{q}_{1_{e}}^{F C}-\bar{q}_{1}^{F C}+\delta\left(\bar{q}_{2_{e}}^{F C}-\bar{q}_{2}^{F C}\right)\right) \\
& =\underline{\tau}_{1}^{F C *}+\delta \underline{\tau}_{2}^{F C *}+2 \delta \Delta \theta\left(\frac{\bar{Z}}{b(1+\delta)}-\frac{2 \Delta \theta \nu_{1}}{b\left(1-\nu_{1}\right)(1+\delta)}\right)
\end{aligned}
$$

Using (29), the tax revenue collected from an inefficient firm without commitment is given by:

$$
\begin{aligned}
\bar{\tau}_{1}^{N C * *} & =\bar{\tau}_{1}^{F C *}-\left(\frac{\delta \bar{Z}}{b(1+\delta)}-\frac{2 \nu_{1} \delta \Delta \theta}{b(1+\delta)\left(1-\nu_{1}\right)}\right)\left(\frac{\delta \bar{Z}}{2(1+\delta)}+\frac{\nu_{1} \Delta \theta}{(1+\delta)\left(1-\nu_{1}\right)}\right) \\
\bar{\tau}_{2}^{N C * *} & =\bar{\tau}_{2}^{F C *}-\left(\frac{\bar{Z}}{b(1+\delta)}-\frac{2 \nu_{1} \Delta \theta}{b(1+\delta)\left(1-\nu_{1}\right)}\right)\left(\frac{\bar{Z}}{2(1+\delta)}+\frac{\delta \nu_{1} \Delta \theta}{(1+\delta)\left(1-\nu_{1}\right)}\right) \\
\bar{\tau}_{1}^{N C * *}+\delta \bar{\tau}_{2}^{N C * *} & =\bar{\tau}_{1}^{F C *}+\delta \bar{\tau}_{2}^{F C *}-\frac{\delta\left(\left(1-\nu_{1}\right) \bar{Z}-2 \Delta \theta \nu_{1}\right)\left(\left(1-\nu_{1}\right) \bar{Z}+2 \Delta \theta \nu_{1}\right)}{2 b(1+\delta)^{2}\left(1-\nu_{1}\right)^{2}}
\end{aligned}
$$

From $S^{b}<S,\left(1-\nu_{1}\right) \bar{Z}-2 \Delta \theta \nu_{1}<0, \underline{\tau}_{1}^{N C * *}+\delta \underline{\tau}_{2}^{N C * *}<\underline{\tau}_{1}^{F C *}+\delta \underline{\tau}_{2}^{F C *}$ and $\bar{\tau}_{1}^{N C * *}+\delta \bar{\tau}_{2}^{N C * *}>\bar{\tau}_{1}^{F C *}+\delta \bar{\tau}_{2}^{F C *}$.

As compared to full commitment, the tax revenue is lower, the tax cut is given by $\frac{\delta\left(2 \Delta \theta \nu_{1}-\bar{Z}\left(1-\nu_{1}\right)\right)^{2}}{2 b(1+\delta)\left(1-\nu_{1}\right)}$.

Implementation. During the first period, the regulator can propose a menu of two contracts. A first contract composed by a royalty $\bar{r}_{1}^{N C * *}=\frac{(1-\delta) \Delta \theta \nu_{1}}{p_{1}\left(1-\nu_{1}\right)}$, a license fee $\bar{L}_{1}^{N C * *}=\bar{\tau}_{1}^{F B}+$ $\frac{\Delta \theta^{2}(1-\delta)^{2}(1+2 \delta) \nu_{1}^{2}}{2 b(1+\delta)^{2}\left(1-\nu_{1}\right)^{2}}+\frac{\Delta \theta \bar{Z}(1-\delta) \delta^{2} \nu_{1}}{b(1+\delta)^{2}\left(1-\nu_{1}\right)}-\frac{\Delta \theta \bar{X}_{1}(1-\delta) \nu_{1}}{b\left(1-\nu_{1}\right)}$, and an upper limit to the extraction $q_{1} \leq$ $\bar{q}_{1_{e}}^{F C}$. A second contract only imposing a license fee $\underline{L}_{1}^{N C * *}=\underline{\tau}_{1}^{F B}-\Delta \theta\left(\frac{\bar{X}_{1}}{b}+\frac{\delta \bar{X}_{2}}{b}-\frac{\Delta \theta(1-\delta)^{2} \nu_{1}}{b(1+\delta)\left(1-\nu_{1}\right)}-\frac{2 \delta \bar{Z}}{b(1+\delta)}\right)$, 
and a minimum quantity to extract $q_{1} \geq \underline{q}_{1_{e}}^{F B}$. If during the first period, the first contract was

chosen, the second-period contract only specifies a license fee $\bar{L}_{2}^{N C * *}=\bar{\tau}_{2}^{F B}-\frac{(1-\delta)^{2} \Delta \theta^{2} \nu_{1}{ }^{2}}{2 b(1+\delta)^{2}\left(1-\nu_{1}\right)^{2}}+$ $\frac{(1-\delta) \bar{Z} \Delta \theta\left(1-\nu_{1}\right) \nu_{1}}{b(1+\delta)^{2}\left(1-\nu_{1}\right)^{2}}$. If during the first period the second contract was chosen, the second-period contract only specifies a license fee $\underline{L}_{2}^{N C * *}=\underline{\tau}_{2}^{F B}$.

\section{Both incentive constraints bind and only an efficient firm exhausts the stock}

\section{D.1 Production}

\section{Full-separating contracts}

If $x=1$ and $y=0, \underline{\nu}_{2}=1 ; \bar{\nu}_{2}=0 ; \bar{q}_{2 / \bar{C}_{1}}=\bar{q}_{2}^{F B}=\frac{\bar{X}_{2}}{b} ; \bar{q}_{2 / \underline{C}_{1}}=0 ;(28) \Leftrightarrow \bar{q}_{1}\left(\underline{q}_{1}\right)=\underline{q}_{1}-\delta \frac{\bar{X}_{2}}{b}$.

Using $\underline{q}_{2}=S-\underline{q}_{1}$, the regulator's payoff becomes:

$$
\begin{aligned}
\max _{\underline{q}_{1}} V_{R}^{N C} & =\nu_{1}\left(\underline{\tau}_{1}^{R}-\Delta \theta \underline{q}_{1}+\delta \underline{\tau}_{2 / \underline{C}_{1}}^{R}\right)+\left(1-\nu_{1}\right)\left(\bar{\tau}_{1}^{R}+\delta \bar{\tau}_{2 / \bar{C}_{1}}^{R}\right) \\
\frac{\partial V_{R}^{N C}}{\partial \underline{q}_{1}} & =\nu_{1}\left(\frac{\partial \underline{\tau}_{1}^{R}}{\partial \underline{q}_{1}}-\Delta \theta+\delta \frac{\partial \underline{\tau}_{2 / \underline{C}_{1}}^{R}}{\partial \underline{q}_{1}}\right)+\left(1-\nu_{1}\right) \frac{\partial \bar{\tau}_{1}^{R}}{\partial \bar{q}_{1}} \frac{\partial \bar{q}_{1}}{\partial \underline{q}_{1}} \\
& =\nu_{1}\left(\underline{X}_{1}-b \underline{q}_{1}-\Delta \theta-\delta\left(\underline{X}_{2}-b\left(S-\underline{q}_{1}\right)\right)\right)+\left(1-\nu_{1}\right)\left(\bar{X}_{1}-b\left(\underline{q}_{1}-\delta \frac{\bar{X}_{2}}{b}\right)\right)
\end{aligned}
$$

Solving with respect to $\underline{q}_{1}$ gives:

$\underline{q}_{1_{e}}^{F S_{1}}=\frac{\left(b \delta S-\left(\bar{X}_{2}+\underline{X}_{2}\right) \delta\right) \nu_{1}+\delta \bar{X}_{2}+\bar{X}_{1}}{b\left(1+\delta \nu_{1}\right)}=\underline{q}_{1_{e}}^{F B}+\frac{\left(1-\nu_{1}\right)}{1+\delta \nu_{1}}\left(\bar{q}_{1}^{F C}+\delta \bar{q}_{2}^{F B}-\underline{q}_{1_{e}}^{F B}\right)$

The modified Hotelling's rule is given by:

$p_{1}-M C\left(\underline{\theta}, \underline{q}_{1}\right)-\Delta \theta+\frac{\left(1-\nu_{1}\right)}{\nu_{1}}\left(p_{1}-M C\left(\bar{\theta}, \bar{q}_{1}\left(\underline{q}_{1}\right)\right)\right)=\delta\left(p_{2}-M C\left(\underline{\theta}, S-\underline{q}_{1}\right)\right) \quad\left(\underline{H R}^{F S_{1}}\right)$

Two opposite effects are at stake. First, the efficient firm's first-period extraction should be distorted downward to decrease the informational rent. Second, due to the countervailing incentives and the induced correlation between the firms' extraction, the efficient firm's first- 
period extraction should be distorted upward. The second effect prevails and an efficient firm produces during the first period more than the first best.

By replacing $\underline{q}_{1_{e}}^{F S 1}$ into $\bar{q}_{1}=\underline{q}_{1}-\delta \frac{\bar{X}_{2}}{b}$, we obtain:

$\bar{q}_{1}^{F S_{1}}=\frac{\bar{X}_{1}-\delta \nu_{1}\left(\bar{X}_{2}(1+\delta)+\underline{X}_{2}-b S\right)}{b\left(1+\delta \nu_{1}\right)}=\bar{q}_{1}^{F C}-\frac{(1+\delta) \nu_{1}}{1+\delta \nu_{1}}\left(\bar{q}_{1}^{F C}+\delta \bar{q}_{1}^{F B}-\underline{q}_{1_{e}}^{F B}\right)$

$\delta \bar{X}_{2}\left(2 \nu_{1}-1\right)+\delta \Delta \theta \nu_{1}+b S-\bar{X}_{1}>0$

$\bar{X}_{1}-\delta \nu_{1}\left(\bar{X}_{2}(2+\delta)+\Delta \theta-b S\right)>0$

$A 4_{a}$ implies that $\underline{q}_{2 e}^{F S_{1}}=S-\underline{q}_{1_{e}}^{F S_{1}}>0$ and $A 4_{b}$ implies that $\bar{q}_{1}^{F S_{1}}>0$.

$\bar{q}_{1}^{F S_{1}}+\bar{q}_{2}^{F B}-S=\frac{\bar{Z}-\left(\delta \bar{X}_{2}+\underline{X}_{2}\right) \delta \nu_{1}}{b\left(1+\delta \nu_{1}\right)}$ defines $S^{d}=\frac{\bar{X}_{1}}{b}+\frac{\bar{X}_{2}}{b}-\frac{\delta \nu_{1}\left(\underline{X}_{2}+\delta \bar{X}_{2}\right)}{b}$.

We now compare the extraction to the full commitment case. Note that $S^{d}$ can be greater or lower than $S^{b}$.

- From $A 3_{a}$ violated, $\underline{q}_{1_{e}}^{F S_{1}}>\underline{q}_{1_{e}}^{F B}$

- If $S>S^{d}>S^{b}$, with commitment, an inefficient firm produces $\bar{q}_{1}^{F C}$. From (49) and $A 3_{a}$ violated, $\bar{q}_{1}^{F S_{1}}<\bar{q}_{1}^{F C}$.

- If $S^{d}<S<S^{b}$, with commitment, an inefficient firm produces $\bar{q}_{1_{e}}^{F C}$.

From $S<S^{b}, \bar{q}_{1 e}^{F C}+\bar{q}_{2_{e}}^{F C}=S$, and from $S>S^{d}, \bar{q}_{1}^{F S_{1}}+\bar{q}_{2}^{F B}<S$. By combining the two, $\bar{q}_{1}^{F S_{1}}+\bar{q}_{2}^{F B}<\bar{q}_{1_{e}}^{F C}+\bar{q}_{2_{e}}^{F C} \Leftrightarrow \bar{q}_{1}^{F S_{1}}-\bar{q}_{1_{e}}^{F C}<\bar{q}_{2_{e}}^{F C}-\bar{q}_{2}^{F B}$.

$\bar{q}_{2_{e}}^{F C}-\bar{q}_{2}^{F B}=-\frac{\bar{Z}\left(1-\nu_{1}\right)-\Delta \theta \nu_{1}(1-\delta)}{b(1+\delta)}$ which is negative since $S<S^{b}$ implies $\bar{Z}\left(1-\nu_{1}\right)-$ $2 \Delta \theta \nu_{1}>0$. Hence, $\bar{q}_{1}^{F S_{1}}<\bar{q}_{1_{e}}^{F C}$

Implementation. The government can propose during the first period, a menu of two contracts. The first contract specifies a royalty on revenue: $\bar{r}_{1}^{F S_{1}}=\frac{\delta \nu_{1}\left(\bar{Z}+\delta \bar{X}_{2}+\underline{X}_{2}\right)}{p_{1}\left(1+\delta \nu_{1}\right)}$, a license fee $\bar{L}_{1}^{F S_{1}}=\frac{\left(\bar{X}_{1}-\delta \nu_{1}\left((1+\delta) \bar{X}_{2}+\underline{X}_{2}-b S\right)\right)^{2}}{2 b\left(1+\delta \nu_{1}\right)^{2}}$, and a maximum quantity to extract: $q_{1} \leq \bar{q}_{1}^{F S_{1}}$, while the second contract specifies a royalty $\underline{r}_{1}^{F S_{1}}=\frac{(1+\delta) \Delta \theta-\delta\left(1-\nu_{1}\right)\left((1+\delta) \bar{X}_{2}+\underline{Z}\right)}{p_{1}\left(1+\delta \nu_{1}\right)}$, a license fee $\underline{L}_{1}^{F S_{1}}=$ 


\section{$\frac{\left(\bar{X}_{1}(1+2 \delta)-\Delta \theta\left(3 \delta \nu_{1}+2(1-\delta)\right)+\delta\left(\bar{X}_{2}\left(2\left(\delta\left(1-\nu_{1}\right)-\nu_{1}\right)+3\right)-b S\left(2-\nu_{1}\right)\right)\right)\left(\delta\left(b S \nu_{1}-\underline{X}_{2} \nu_{1}+\bar{X}_{2}\left(1-\nu_{1}\right)\right)+\bar{X}_{1}\right)}{2 b\left(1+\delta \nu_{1}\right)^{2}}$, and a} minimum quantity to extract $q_{1} \geq \underline{q}_{1}^{F B}$. If during the first period the first contract was chosen, the second-period contract only specifies a license fee $\bar{L}_{2}^{F S_{1}}=\frac{\bar{X}_{2}^{2}}{2 b}$. If during the first period the second contract was chosen, the second-period contract only specifies a license fee $\underline{L}_{2}^{F S_{1}}=\frac{\left(\Delta \theta \delta \nu_{1}+\bar{X}_{2}(1+\delta)+\underline{Z}\right)\left(\bar{X}_{2}\left(2 \delta \nu_{1}+1-\delta\right)+\Delta \theta\left(2+\delta \nu_{1}\right)-\underline{Z}\right)}{2 b\left(1+\delta \nu_{1}\right)^{2}}$.

\section{Semi-separating contracts}

If $x=1$ and $y>0$ implies: $\underline{\nu}_{2}=\frac{\nu_{1}}{\nu_{1}+\left(1-\nu_{1}\right) y} ; \bar{\nu}_{2}=0 ; \bar{q}_{2 / \bar{C}_{1}}=\bar{q}_{2}^{F B}=\frac{\bar{X}_{2}}{b} ; \bar{q}_{2 / C_{1}}=\frac{\bar{X}_{2}}{b}-\frac{\Delta \theta \nu_{1}}{b\left(1-\nu_{1}\right) y}$. $(28) \Leftrightarrow \bar{q}_{1}=\underline{q}_{1}-\delta \frac{\Delta \theta \nu_{1}}{b\left(1-\nu_{1}\right) y}$. Using $\underline{q}_{2}=S-\underline{q}_{1}$ and $\bar{q}_{1}=\underline{q}_{1}-\frac{\delta \Delta \theta \nu_{1}}{b\left(1-\nu_{1}\right) y}$, the regulator regulator's payoff becomes:

$$
\begin{aligned}
& \max _{\underline{q}_{1}} V_{R}^{N C}=\left(\nu_{1}+\left(1-\nu_{1}\right) y\right)\left(\underline{\tau}_{1}^{R}-\Delta \theta \underline{q}_{1}\right)+\left(1-\nu_{1}\right)(1-y) \bar{\tau}_{1}^{R}+\delta\left(1-\nu_{1}\right)\left((1-y) \bar{\tau}_{2 / \bar{C}_{1}}^{R}+y \bar{\tau}_{2 / \underline{C}_{1}}^{R}\right) \\
& +\delta \nu_{1}\left(\underline{\tau}_{2 / \underline{C}_{1}}^{R}-\Delta \theta\left(\frac{\bar{X}_{2}}{b}-\frac{\Delta \theta \nu_{1}}{b\left(1-\nu_{1}\right) y}\right)\right) \\
& \frac{\partial V_{R}^{N C}}{\partial \underline{q}_{1}}=\left(\nu_{1}+\left(1-\nu_{1}\right) y\right)\left(\frac{\partial \underline{\tau}_{1}^{R}}{\partial \underline{q}_{1}}-\Delta \theta\right)+\left(1-\nu_{1}\right)(1-y) \frac{\partial \bar{\tau}_{1}^{R}}{\partial \bar{q}_{1}} \frac{\partial \bar{q}_{1}}{\partial \underline{q}_{1}} \\
& +\delta\left(1-\nu_{1}\right)\left((1-y) \frac{\partial \bar{\tau}_{2 / \bar{C}_{1}}^{R}}{\partial \underline{q}_{1}}+y \frac{\partial \bar{\tau}_{2 / \underline{C}_{1}}^{R}}{\partial \underline{q}_{1}}\right) \frac{\partial \bar{q}_{1}}{\partial \underline{q}_{1}}+\delta \nu_{1} \frac{\partial \underline{\tau}_{2 / \underline{C}_{1}}^{R}}{\partial \underline{q}_{1}} \\
& =\left(\nu_{1}+\left(1-\nu_{1}\right) y\right)\left(\underline{X}_{1}-b \underline{q}_{1}-\Delta \theta\right)+\left(1-\nu_{1}\right)(1-y)\left(\bar{X}_{1}-b\left(\underline{q}_{1}-\frac{\delta \Delta \theta \nu_{1}}{b\left(1-\nu_{1}\right) y}\right)\right) \\
& -\delta \nu_{1}\left(\underline{X}_{2}-b\left(S-\underline{q}_{1}\right)\right)
\end{aligned}
$$

By solving, we obtain:

$\underline{q}_{1}(y)=\frac{\left(\bar{X}_{1}-\delta\left(\underline{X}_{2}-b S\right) \nu_{1}\right) y+\Delta \theta \delta \nu_{1}(1-y)}{b\left(1+\delta \nu_{1}\right) y}$

We replace $\underline{q}_{1}(y)$ into $V_{R}^{N C}$ and we differentiate and solve with respect to $y$. This gives:

$y^{*}=\frac{2 \delta(\delta+1) \Delta \theta \nu_{1}}{\Delta \theta\left(\delta^{2} \nu_{1}+5 \delta \nu_{1}-3 \delta+1\right)-2 \delta \bar{Z}\left(1-\nu_{1}\right)}$ 
$y^{*}<1$ ensures that $y^{*}>0 . y^{*}<1$, if $A 5_{a}$ is satisfied where:

$\Delta \theta\left(1-\delta^{2} \nu_{1}-3 \delta\left(1-\nu_{1}\right)\right)-2 \delta\left(\bar{X}_{1}+\bar{X}_{2}-b S\right)\left(1-\nu_{1}\right)>0$

By replacing $y^{*}$ into (51), the modified Hotelling's rule is given by:

$p_{1}-M C\left(\underline{\theta}, \bar{q}_{1}\right)-\frac{(1+\delta) \Delta \theta}{2}=\delta\left(p_{2}-M C\left(\underline{\theta}, S-\bar{q}_{1}\right)\right)$

From the modified Hotelling's rule, as compared to the first best, the efficient firm's first-period production is distorted downward.

By replacing $y^{*}$ into $\underline{q}_{1}(y)$ and $\bar{q}_{2 / \underline{C}_{1}}$ we obtain:

$$
\begin{aligned}
\underline{q}_{1_{e}}^{S S_{y}} & =\underline{q}_{1_{e}}^{F B}-\frac{\Delta \theta}{2 b} \\
\bar{q}_{2}^{S S_{y}}\left(\underline{\nu}_{2}\right) & =\bar{q}_{2}^{F C}-\frac{\Delta \theta(1-\delta)\left(1+\delta \nu_{1}\right)}{2 b \delta\left(1-\nu_{1}\right)(1+\delta)}+\frac{\bar{Z}+\Delta \theta}{b(1+\delta)}
\end{aligned}
$$

We then replace $\underline{q}_{1_{e}}^{S S y}$ and $y^{*}$ into $\bar{q}_{1}=\underline{q}_{1}-\frac{\delta \Delta \theta \nu_{1}}{b\left(1-\nu_{1}\right) y}$ and we obtain:

$\bar{q}_{1}^{S S_{y}}=\bar{q}_{1_{e}}^{F B}-\frac{(1+\delta)^{2} \nu_{1} \Delta \theta-2 \delta\left(1-\nu_{1}\right) \bar{Z}}{2 b\left(1-\nu_{1}\right)(1+\delta)}=\bar{q}_{1}^{F C}+\frac{(1-\delta) \nu_{1} \Delta \theta}{2 b\left(1-\nu_{1}\right)}$

$\underline{q}_{1 e}^{S S y}>0, \bar{q}_{1}^{S S y}>0$, and $\bar{q}_{2}^{S S y}\left(\underline{\nu}_{2}\right)>0$ are satisfied if $A 5_{b}, A 5_{c}$, and $A 5_{d}$ are respectively satisfied, where:

$$
\begin{aligned}
& (1-3 \delta) \Delta \theta+2\left(\bar{X}_{1}-\delta \bar{X}_{2}-\delta b S\right)>0 \\
& 2 \bar{X}_{1}\left(1-\nu_{1}\right)-(1+\delta) \Delta \theta \nu_{1}>0 \\
& 2 \delta\left((2+\delta) \bar{X}_{2}+\bar{X}_{1}-b S\right)-\Delta \theta\left(\delta^{2} \nu_{1}+5 \delta \nu_{1}+1-3 \delta\right) \\
& \bar{q}_{1}^{S S y}+\bar{q}_{2}^{F B}-S=\frac{\bar{X}_{1}}{b}+\frac{\bar{X}_{2}}{b}-\frac{(1+\delta) \nu_{1} \Delta \theta}{2 b\left(1-\nu_{1}\right)}-S=\frac{2 \bar{Z}\left(1-\nu_{1}\right)-\Delta \theta \nu_{1}(1+\delta)}{2 b\left(1-\nu_{1}\right)} \text { defines } \\
& S^{e}=\frac{\bar{X}_{1}}{b}+\frac{\bar{X}_{2}}{b}-\frac{(1+\delta) \Delta \theta \nu_{1}}{2 b\left(1-\nu_{1}\right)} \text { with } S^{c}<S^{e}<S^{a} .
\end{aligned}
$$

We now compare the extraction to the full commitment case: 
- From $\Delta \theta>0, \underline{q}_{1_{e}}^{S S_{y}}<\underline{q}_{1_{e}}^{F B}$

- Since $S>S^{e}>S^{b}$, with commitment an efficient firm produces $\bar{q}_{1}^{F C}$, from (54) it is clear that $\bar{q}_{1}^{S S y}>\bar{q}_{1}^{F C}$.

- $S>S^{e}$ implies that $(1+\delta) \nu_{1} \Delta \theta-2 \bar{Z}\left(1-\nu_{1}\right)>0$, since $\bar{Z}>0\left(\right.$ from $\left.S<S^{a}\right), \delta<1$, $S>S^{e}$ also implies that $(1+\delta)^{2} \nu_{1} \Delta \theta-2 \delta \bar{Z}\left(1-\nu_{1}\right)>0$ which implies that $\bar{q}_{1}^{S S y}<\bar{q}_{1_{e}}^{F B}$.

Note that the sign of $S^{e}-S^{d}$ is given by the sign of: $2 \bar{X}_{2} \delta(1+\delta)\left(1-\nu_{1}\right)-\left(1-\delta+2 \delta \nu_{1}\right) \Delta \theta$, which is equal to:

$-\left(1-\delta+2 \delta \nu_{1}\right) \Delta \theta+\delta\left(1-\nu_{1}\right)\left(\bar{X}_{1}+(2+\delta) \bar{X}_{2}-b S\right)+\delta\left(1-\nu_{1}\right)\left(\delta \bar{X}_{2}+b S-\bar{X}_{1}\right)$

which is positive from $A 3_{a}$ violated and $A 1_{b}$ satisfied.

\section{Pooling contract}

$(\overline{I C})$ binding implies that $\tau_{1}=\bar{\tau}_{1}^{R}$. Using $\underline{q}_{2}=S-q_{1}$ and $\bar{q}_{2}=\frac{\bar{X}_{2}}{b}-\frac{\Delta \theta \nu_{1}}{b\left(1-\nu_{1}\right)}$, the regulator's payoff becomes:

$$
\begin{aligned}
\max _{q_{1}} V_{R}^{N C} & =\bar{\tau}_{1}^{R}+\delta \nu_{1}\left(\underline{\tau}_{2}^{R}-\Delta \theta\left(\frac{\bar{X}_{2}}{b}-\frac{\Delta \theta \nu_{1}}{b\left(1-\nu_{1}\right)}\right)\right)+\delta\left(1-\nu_{1}\right) \bar{\tau}_{2}^{R} \\
\frac{\partial V_{R}^{N C}}{\partial q_{1}} & =\frac{\partial \bar{\tau}_{1}^{R}}{\partial q_{1}}+\delta \nu_{1} \frac{\partial \underline{\tau}_{2}^{R}}{\partial q_{1}}=\left(\bar{X}_{1}-b q_{1}\right)-\delta \nu_{1}\left(\underline{X}_{2}-b\left(S-q_{1}\right)\right)=0
\end{aligned}
$$

The modified Hotelling's rule is given by:

$p_{1}-M C\left(\underline{\theta}, \underline{q}_{1}\right)-\Delta \theta=\delta \nu_{1}\left(p_{2}-M C\left(\underline{\theta}, S-\underline{q}_{1}\right)\right)$

Two opposite effects are at stake. The first-period production is lower than the efficient firm's first-best level since the contract is also designed for an inefficient firm. However, since only an efficient firm exhausts the stock, the opportunity cost of extracting during the first period is lower than under symmetric. This second effect tends to increase the first-period production above an efficient firm's first-best level. Nevertheless, the first effect prevails and under a pooling contract the first-period production is lower than the efficient firm's first-best level. 
Solving the first-order condition gives:

$$
\begin{aligned}
q_{1}^{P_{1}} & =\bar{q}_{1_{e}}^{F B}-\frac{\delta\left((1+\delta) \nu_{1} \Delta \theta-\bar{Z}\left(1-\nu_{1}\right)\right)}{b\left(1+\delta \nu_{1}\right)(1+\delta)}=\underline{q}_{1_{e}}^{F B}-\frac{\left(1-\delta+2 \delta \nu_{1}\right) \Delta \theta-\delta \bar{Z}\left(1-\nu_{1}\right)}{b\left(1+\delta \nu_{1}\right)(1+\delta)} \\
& =\bar{q}_{1}^{F C}+\frac{\nu_{1}\left(\left(1-\delta+2 \delta \nu_{1}\right) \Delta \theta-\delta\left(1-\nu_{1}\right) \bar{Z}\right)}{b\left(1+\delta \nu_{1}\right)\left(1-\nu_{1}\right)} \\
q_{1}^{P_{1}} & >0 \text { if } \bar{X}_{1}-\delta \nu_{1}\left(\bar{X}_{2}+\Delta \theta-b S\right)>0 . \\
\bar{q}_{1}^{P_{1}} & +\bar{q}_{2}^{F C}-S=\frac{\bar{Z}\left(1-\nu_{1}\right)-\Delta \theta \nu_{1}(1+\delta)}{b\left(1-\nu_{1}\right)\left(1+\delta \nu_{1}\right)} \text { defines } S^{f}=\frac{\bar{X}_{1}}{b}+\frac{\bar{X}_{2}}{b}-\frac{(1+\delta) \Delta \theta \nu_{1}}{b\left(1-\nu_{1}\right)} .
\end{aligned}
$$

We now compare the extraction to the full commitment case:

- $S>S^{f}$ implies that $\Delta \theta \nu_{1}(1+\delta)-\bar{Z}\left(1-\nu_{1}\right)>0$ which implies that $q_{1}^{P_{1}}<\bar{q}_{1_{e}}^{F B}<\underline{q}_{1_{e}}^{F B}$. Moreover, $\bar{q}_{1}^{P_{1}}>\bar{q}_{1}^{F C}$, indeed,

$$
\left(1-\delta+2 \delta \nu_{1}\right) \Delta \theta-\delta\left(1-\nu_{1}\right) \bar{Z}=(1+\delta) \nu_{1} \Delta \theta-\bar{Z}\left(1-\nu_{1}\right)+(\bar{Z}+\Delta \theta)(1-\delta)\left(1-\nu_{1}\right)
$$

which is positive from $S>S^{f}$.

$S^{b}<S^{f}<S^{c}$, indeed, under full pooling, the inefficient firm's first-period extraction is larger than the one with commitment, while the second period-extraction is the same. Moreover, an inefficient firm produces during the second period less under a pooling contract than under full separation. This lower production implies that the stock may not be exhausted by an inefficient firm under a pooling contract, while it is exhausted with full separation and no countervailing incentives.

Implementation. The government can propose during the first period one contract that specifies a royalty on revenue $r_{1}^{P_{1}}=\frac{(\underline{Z}-\Delta \theta) \delta \nu_{1}}{p_{1}\left(1+\delta \nu_{1}\right)}$, a license fee $L_{1}^{P_{1}}=\frac{\left(\bar{X}_{1}-\delta\left(\underline{X}_{2}-b S\right)\right)^{2}}{2 b\left(1+\delta \nu_{1}\right)^{2}}$, and a maximum quantity to extract: $q_{1} \leq q_{1}^{P_{1}}$. During the second period, a menu of two contract is proposed, the first contract specifies a royalty on the revenue $\bar{r}_{2}^{F C}$ (defined previously) and a license fee $\bar{L}_{2}^{P_{1}}=\frac{\left(\bar{X}_{2}-\underline{X}_{2} \nu_{1}\right)^{2}}{2 b\left(1-\nu_{1}\right)^{2}}$. The second contract only specifies a license fee:

$\underline{L}_{2}^{P_{1}}=\bar{\tau}_{2}^{F B}+\frac{\left(\Delta \theta(1+\delta) \nu_{1}-\bar{Z}\left(1-\nu_{1}\right)\right)\left(\Delta \theta(1+\delta)\left(\left(1+\delta \nu_{1}\right)^{2}+\delta^{2} \nu_{1}+2 \delta+1\right)+\bar{Z} \delta\left(1-\nu_{1}\right)\left(\delta \nu_{1}+\delta+2\right)\right)}{2 b(1+\delta)^{2}\left(1-\nu_{1}\right)\left(1+\delta \nu_{1}\right)^{2}}$. 


\section{D.2 Tax revenue}

We now define the conditions under which each contract is proposed:

$$
\begin{aligned}
V^{F S_{1}}-V^{P_{1}} & =\frac{\bar{X}_{2} \delta \nu_{1}\left(\left(2 \Delta \theta-\delta \bar{X}_{2}\left(1-\nu_{1}\right)\right)(1+\delta)-2 \delta \underline{Z}\left(1-\nu_{1}\right)\right)}{2 b\left(1+\delta \nu_{1}\right)}-\frac{\delta \Delta \theta^{2} \nu_{1}^{2}}{2 b\left(1-\nu_{1}\right)} \\
V^{F S_{1}}-V^{S S y} & =\frac{\bar{X}_{2} \delta \nu_{1}\left(\left(2 \Delta \theta-\delta \bar{X}_{2}\left(1-\nu_{1}\right)\right)(1+\delta)-2 \delta \underline{Z}\left(1-\nu_{1}\right)\right)}{2 b\left(1+\delta \nu_{1}\right)}-\frac{\delta \Delta \theta^{2} \nu_{1}^{2}}{2 b\left(1-\nu_{1}\right)} \\
& -\frac{\nu_{1}\left(2 \delta \underline{Z}\left(1-\nu_{1}\right)-(1+\delta) \Delta \theta\left(1-\delta \nu_{1}\right)\right)^{2}}{8 b(1+\delta)\left(1-\nu_{1}\right)\left(1+\delta \nu_{1}\right)} \\
V^{S S y}-V^{P_{1}} & =\frac{\nu_{1}\left(2 \delta \underline{Z}\left(1-\nu_{1}\right)-(1+\delta) \Delta \theta\left(1-\delta \nu_{1}\right)\right)^{2}}{8 b(1+\delta)\left(1-\nu_{1}\right)\left(1+\delta \nu_{1}\right)}
\end{aligned}
$$

A pooling contract is proposed if $\delta>\delta^{P_{1}}$, where $\delta^{P_{1}}$ is given by:

$\bar{X}_{2}\left(\left(2 \Delta \theta-\delta^{P_{1}} \bar{X}_{2}\left(1-\nu_{1}\right)\right)\left(1+\delta^{P_{1}}\right)-2 \delta^{P_{1}} \underline{Z}\left(1-\nu_{1}\right)\right)\left(1-\nu_{1}\right)-\Delta \theta^{2} \nu_{1}\left(\delta^{P_{1}} \nu_{1}+1\right)=0$

A semi-separating contract is proposed if $\delta>\delta^{S S_{y}}$ where $\delta^{S S_{y}}$ is given by:

$$
\begin{aligned}
& -\left(2 \delta^{S S_{y}} \underline{Z}\left(1-\nu_{1}\right)-\left(1+\delta^{S S_{y}}\right) \Delta \theta\left(1-\delta^{S S_{y}} \nu_{1}\right)\right)^{2}-4 \nu_{1} \Delta \theta^{2} \delta^{S S_{y}}\left(1+\delta^{S S_{y}}\right)\left(\delta^{S S_{y}} \nu_{1}+1\right) \\
& \quad+4 \bar{X}_{2} \delta^{S S_{y}}\left(1+\delta^{S S_{y}}\right)\left(1-\nu_{1}\right)\left(\left(2 \Delta \theta-\delta^{S S_{y}} \bar{X}_{2}\left(1-\nu_{1}\right)\right)\left(1+\delta^{S S_{y}}\right)-2 \delta^{S S_{y}} \underline{Z}\left(1-\nu_{1}\right)\right)=0
\end{aligned}
$$

\section{E Proof of Proposition 2}

\section{E.1 Production}

\section{Full-separating contracts}

If $x=1$ and $y=0, \underline{\nu}_{2}=1 ; \bar{\nu}_{2}=0 ; \bar{q}_{2 / \underline{C}_{1}}=0 ;(28) \Leftrightarrow \underline{q}_{1}\left(\bar{q}_{1}\right)=\bar{q}_{1}+\delta\left(S-\bar{q}_{1}\right)$.

Using $\underline{q}_{1}\left(\bar{q}_{1}\right), \underline{q}_{2}=S-\underline{q}_{1}=(1-\delta)\left(S-\bar{q}_{1}\right)$, and $\bar{q}_{2 / \bar{C}_{1}}=S-\bar{q}_{1}$, the regulator's payoff only 
depends on $\bar{q}_{1}$ :

$$
\begin{aligned}
& \max _{\bar{q}_{1}} V_{R}^{N C}=\nu_{1}\left(\underline{\tau}_{1}^{R}-\Delta \theta \underline{q}_{1}\left(\bar{q}_{1}\right)+\delta \underline{\tau}_{2 / \underline{C}_{1}}^{R}\right)+\left(1-\nu_{1}\right)\left(\bar{\tau}_{1}^{R}+\delta \bar{\tau}_{2 / \bar{C}_{1}}^{R}\right) \\
\frac{\partial V_{R}^{N C}}{\partial \bar{q}_{1}} & =\nu_{1}\left(\frac{\partial \underline{\tau}_{1}^{R}}{\partial \underline{q}_{1}}-\Delta \theta+\delta \frac{\partial \underline{\tau}_{2 / \underline{C}_{1}}^{R}}{\partial \underline{q}_{1}}\right) \frac{\partial \underline{q}_{1}}{\partial \bar{q}_{1}}+\left(1-\nu_{1}\right)\left(\frac{\partial \bar{\tau}_{1}^{R}}{\partial \bar{q}_{1}}+\delta \frac{\partial \bar{\tau}_{2 / \bar{C}_{1}}^{R}}{\partial \bar{q}_{1}}\right) \\
& =\nu_{1}\left(\underline{X}_{1}-b \underline{q}_{1}\left(\bar{q}_{1}\right)-\Delta \theta-\delta\left(\underline{X}_{2}-b\left(S-\underline{q}_{1}\left(\bar{q}_{1}\right)\right)\right)\right)(1-\delta) \\
& +\left(1-\nu_{1}\right)\left(\bar{X}_{1}-b \bar{q}_{1}-\delta\left(\bar{X}_{2}-b\left(S-\bar{q}_{1}\right)\right)\right)
\end{aligned}
$$

Solving this problem gives:

$\bar{q}_{1_{e}}^{F S}=\bar{q}_{1_{e}}^{F B}-\frac{(1-\delta) \delta \nu_{1}\left(\delta \bar{X}_{2}+\underline{X}_{2}-\bar{Z}\right)}{b(1+\delta)\left(1-(2-\delta) \delta \nu_{1}\right)}=\bar{q}_{1_{e}}^{F C}-\frac{(1-\delta) \nu_{1}}{1-(2-\delta) \delta \nu_{1}}\left(\bar{q}_{1_{e}}^{F C}+\delta\left(S-\bar{q}_{1_{e}}^{F C}\right)-\underline{q}_{1_{e}}^{F B}\right)$

Substituting $\bar{q}_{1_{e}}^{F S}$ in $\underline{q}_{1}=\bar{q}_{1}+\delta\left(S-\bar{q}_{1}\right)$ gives:

$\underline{q}_{1_{e}}^{F S}=\underline{q}_{1_{e}}^{F B}+\frac{\left(1-\nu_{1}\right)}{1-(2-\delta) \delta \nu_{1}}\left(\bar{q}_{1_{e}}^{F C}+\delta\left(S-\bar{q}_{1_{e}}^{F C}\right)-\underline{q}_{1_{e}}^{F B}\right)$

$A 6_{b}$ implies that $S-\underline{q}_{1_{e}}^{F S}>0, A 6_{c}$ implies that $\bar{q}_{1_{e}}^{F S}>0$.

$\left(1-\delta \nu_{1}\right)\left(\delta \underline{X}_{2}-\underline{X}_{1}+b S\right)+(1-\delta) \Delta \theta>0$

$b S(1+\delta)\left(1-(2-\delta) \delta \nu_{1}\right)-\left(1-\delta \nu_{1}\right)\left(\delta \underline{X}_{2}-\underline{X}_{1}+b S\right)-(1-\delta) \Delta \theta>0$

The modified Hotelling's rule for an efficient firm is given by:

$$
\begin{aligned}
& p_{1}-M C\left(\underline{\theta}, \underline{q}_{1}\right)-\Delta \theta+\frac{1-\nu_{1}}{\nu_{1}(1-\delta)}\left(p_{1}-M C\left(\bar{\theta}, \bar{q}_{1}\left(\underline{q}_{1}\right)\right)\right) \\
& =\delta\left(p_{2}-M C\left(\underline{\theta}, S-\underline{q}_{1}\right)\right)+\frac{\delta\left(1-\nu_{1}\right)}{\nu_{1}(1-\delta)}\left(p_{2}-M C\left(\bar{\theta}, S-\bar{q}_{1}\left(\underline{q}_{1}\right)\right)\right)
\end{aligned}
$$

Two opposite effects are at stake. The informational rent decreases an efficient firm first-period extraction. Indeed, an increase in $\underline{q}_{1}$ increases the informational rent by $\Delta \theta$. However, the production correlation calls for an upward distortion. Indeed, the efficient firm's first-period production should increase to increase the inefficient firm's first-period extraction. This second 
effect prevails and an efficient firm produces more than the first best.

The modified Hotelling's rule for an inefficient firm is given by:

$$
\begin{aligned}
& p_{1}-M C\left(\bar{\theta}, \bar{q}_{1}\right)-\frac{\Delta \theta \nu_{1}}{1-\nu_{1}}+\frac{(1-\delta) \nu_{1}}{1-\nu_{1}}\left(p_{1}-M C\left(\underline{\theta}, \underline{q}_{1}\left(\bar{q}_{1}\right)\right)\right) \\
& =\delta\left(p_{2}-M C\left(\bar{\theta}, S-\bar{q}_{1}\right)-\frac{\Delta \theta \nu_{1}}{1-\nu_{1}}\right)+\frac{\delta(1-\delta) \nu_{1}}{1-\nu_{1}}\left(p_{2}-M C\left(\underline{\theta}, S-\underline{q}_{1}\left(\bar{q}_{1}\right)\right)\right) \quad\left(\overline{H R}^{F S}\right)
\end{aligned}
$$

The informational rent decreases an inefficient firm's first-period extraction. Moreover, the production correlation also calls for a downward distortion. The two effects cumulate and an inefficient firm produces during the first period less than with symmetric information and even less than with commitment.

We now compare the extraction to the full commitment case.

- From $A 3_{b}$ violated, $\underline{q}_{1_{e}}^{F S}>\underline{q}_{1_{e}}^{F B}$.

- If $S<S^{b}$, with commitment the production is $\bar{q}_{1 e}^{F C}$, from $A 3_{b}$ violated, $\bar{q}_{1_{e}}^{F S}<\bar{q}_{1_{e}}^{F C}$ and from $\bar{q}_{1_{e}}^{F C}<\bar{q}_{1_{e}}^{F B}, \bar{q}_{1_{e}}^{F S}<\bar{q}_{1_{e}}^{F B}$. If $S>S^{b}$, with commitment the production is $\bar{q}_{1}^{F C}$. From $\bar{q}_{1_{e}}^{F C}<\bar{q}_{1}^{F C}$ and $\bar{q}_{1_{e}}^{F S}<\bar{q}_{1_{e}}^{F C}, \bar{q}_{1_{e}}^{F S}<\bar{q}_{1}^{F C}$

Hotelling's rule may not hold, to prove this point we take two examples ${ }^{7}$

If $\left(b, S, p_{1}, p_{2}, \nu_{1}, \underline{\theta}, \bar{\theta}\right)=(1,10,20,22, .6,1,4)$, full-separating contracts where both $(I C)$ bind are offered for $\delta \in\left[\delta_{1}, \delta_{2}\right]$ where $\delta_{1}=\frac{5}{9}$ and $\delta_{2}=.6399$. $\frac{\partial \underline{q}_{1}^{F S}}{\partial \delta}<0$ for $\delta \in\left[\frac{5}{9}, .6103\right]$ and $\frac{\partial \underline{q}_{1}^{F S}}{\partial \delta}>0$ for $\delta \in[.6103, .6399]$. $\frac{\partial \bar{q}_{1}^{F S}}{\partial \delta}<0$ for $\delta \in\left[\frac{5}{9}, .6399\right]$.

If $\left(b, S, p_{1}, p_{2}, \nu_{1}, \underline{\theta}, \bar{\theta}\right)=(1,4,10,7, .8, .1,2)$, full-separating contracts where both $(I C)$ bind are offered for $\delta \in\left[\delta_{1}, \delta_{2}\right]$ where $\delta_{1}=\frac{131-3 \sqrt{809}}{52}=0.8783$ and $\delta_{2}=.919438$. $\frac{\partial \bar{q}_{1}^{F S}}{\partial \delta}<0$ for $\delta \in[0.8783,0.8885]$, and $\frac{\partial \bar{q}_{1}^{F S}}{\partial \delta}>0$ for $\delta \in[0.8885, .919438]$. For $\delta \in[0.8783, .919438], \frac{\partial \underline{q}_{1}^{F S}}{\partial \delta}<0$.

Implementation. The government can propose during the first period, a menu of two contracts. The first contract specifies a royalty on revenue $\bar{r}_{1}^{F S}=\frac{\delta(1-\delta)\left(\bar{X}_{2} \delta-\bar{Z}+\underline{X}_{2}\right) \nu_{1}}{p_{1}\left(1-(2-\delta) \delta \nu_{1}\right)}$, a license fee to capture the revenue $\bar{L}_{1}^{F S}$, and a maximum quantity to extract $q_{1} \leq \bar{q}_{1}^{F S}$. The second contract specifies a negative royalty (or a production bonus) $\underline{r}_{1}^{F S}=\frac{\Delta \theta(1-\delta)^{2}+\delta\left(1-\nu_{1}\right)\left(\underline{Z}-(1+\delta) \underline{X}_{2}\right)}{p_{1}\left(1-(2-\delta) \delta \nu_{1}\right)}$,

\footnotetext{
${ }^{7} \delta_{2}$ is the threshold above which a pooling contract is proposed. This threshold is defined in the next subsection.
} 
a license fee $\underline{L}_{1}^{F S}$ to capture the revenue net of the informational rent $\Delta \theta \underline{q}_{1 e}^{F S}$, and a minimum quantity to extract $q_{1} \geq \underline{q}_{1_{e}}^{F B}$. From $\left(A 3_{b}\right)$ violated, it is straightforward that $\underline{r}_{1}^{F S}<0$. During the second period, depending on the contract chosen, only one contract is proposed. This contract only specifies a license fee to capture the entire revenue. For this case, we do not define the license fee since using our functional forms the expressions are long.

\section{Pooling contract}

From (16) bind binding, $\tau_{1}=\bar{\tau}_{1}^{R}$. Using, $\underline{q}_{2}=\bar{q}_{2}=S-q_{1}$, the problem is:

$$
\begin{aligned}
\max _{q_{1}} V_{R}^{P} & =\bar{\tau}_{1}^{R}+\delta\left(\nu_{1}\left(\underline{\tau}_{2}^{R}-\Delta \theta\left(S-q_{1}\right)\right)+\left(1-\nu_{1}\right) \bar{\tau}_{2}^{R}\right) \\
\frac{\partial V_{R}^{P}}{\partial q_{1}} & =\frac{\partial \bar{\tau}_{1}^{R}}{\partial q_{1}}+\delta\left(\nu_{1}\left(\frac{\partial \underline{\tau}_{2}^{R}}{\partial q_{1}}+\Delta \theta\right)+\left(1-\nu_{1}\right) \frac{\partial \bar{\tau}_{2}^{R}}{\partial q_{1}}\right)=0 \\
& \bar{X}_{1}-b q_{1}-\delta\left(\nu_{1}\left(\underline{X}_{2}-b\left(S-q_{1}\right)-\Delta \theta\right)+\left(1-\nu_{1}\right)\left(\bar{X}_{2}-b\left(S-q_{1}\right)\right)\right)=0 \\
\Leftrightarrow & \bar{X}_{1}-b q_{1}-\delta\left(\bar{X}_{1}-b\left(S-q_{1}\right)\right)=0 \Leftrightarrow q_{1}^{P}=\bar{q}_{1_{e} B}^{F B}
\end{aligned}
$$

\section{Conditions under which the stock is exhausted:}

The stock is exhausted if $S<\operatorname{Min}\left[S^{f}, S^{d}\right]$, or if $S^{d}<S<S^{f}$ and $V_{R}^{P}>V_{R}^{F S_{1}}$, or if $S^{f}<S<S^{d}$ and $V_{R}^{F S}>V_{R}^{P_{1}}$, where:

$$
\begin{aligned}
V_{R}^{F S_{1}}-V_{R}^{P} & =\frac{\Delta \theta \delta \nu_{1}\left(2\left(\bar{X}_{2}\left(2 \delta \nu_{1}+1-\delta\right)-\bar{Z}\right)+\Delta \theta \delta \nu_{1}\right)}{2 b\left(1+\delta \nu_{1}\right)} \\
& +\frac{\delta\left(1-\nu_{1}\right)\left(\bar{Z}^{2}-\bar{X}_{2} \delta(\delta+1)\left(\bar{X}_{2}(\delta+1)+2 \bar{Z}\right) \nu_{1}\right)}{2 b(\delta+1)\left(1+\delta \nu_{1}\right)} \\
V_{R}^{P_{1}} & =\frac{\bar{X}_{1}^{2}}{2 b}-\frac{(\bar{Z}+\Delta \theta)^{2} \delta \nu_{1}}{2 b\left(\delta \nu_{1}+1\right)}+\frac{\delta\left(\Delta \theta^{2} \nu_{1}+\bar{X}_{2}^{2}\left(1-\nu_{1}\right)\right)}{2 b\left(1-\nu_{1}\right)}
\end{aligned}
$$




$$
\begin{aligned}
V_{R}^{F S} & =\left(\frac{\underline{X}_{1}^{2}}{2 b}+\delta \frac{\underline{X}_{2}^{2}}{2 b}-\frac{\underline{Z}^{2} \delta}{2 b(\delta+1)}\right) \nu_{1}+\left(\frac{\bar{X}_{1}^{2}}{2 b}+\delta \frac{\bar{X}_{2}^{2}}{2 b}-\frac{\bar{Z}^{2} \delta}{2 b(\delta+1)}\right)\left(1-\nu_{1}\right) \\
& -\frac{\nu_{1}\left(a \delta\left(\nu_{1}-1\right)-\Delta \theta\left(\delta^{2} \nu_{1}-2 \delta \nu_{1}+1\right)\right)^{2}}{2 b(\delta+1)\left(\delta^{2} \nu_{1}-2 \delta \nu_{1}+1\right)^{2}}-\frac{a^{2}(\delta-1)^{2} \delta^{2}\left(1-\nu_{1}\right) \nu_{1}^{2}}{2 b(\delta+1)\left(\delta^{2} \nu_{1}-2 \delta \nu_{1}+1\right)^{2}} \\
& -\frac{\Delta \theta \nu_{1}\left(a(1-\delta)\left(1-\delta \nu_{1}\right)\right)}{b(\delta+1)\left(\delta^{2} \nu_{1}-2 \delta \nu_{1}+1\right)}-\frac{\Delta \theta \nu_{1}(b(1+\delta) S+\Delta \theta(1-\delta))}{b(\delta+1)\left(\delta^{2} \nu_{1}-2 \delta \nu_{1}+1\right)}
\end{aligned}
$$

where $a=\bar{X}_{1}-\delta \bar{X}_{2}-b S-\Delta \theta$

Implementation. The government can propose during the first period one contract that only specifies a license fee $L_{1}^{P}=\bar{\tau}_{1}^{F B}$, and a maximum quantity to extract: $q_{1} \leq \bar{q}_{1_{e}}^{F B}$. During the second period, a menu of two contract is proposed, the first contract specifies the royalty on the revenue $\bar{r}_{2}^{F C}$ and a license fee $\bar{L}_{2}^{P}=\bar{\tau}_{2}^{F B}-\frac{\Delta \theta \nu_{1}}{1-\nu_{1}}\left(\frac{\bar{X}_{2}}{b}-\frac{\bar{Z}}{b(1+\delta)}\right)$. The second contract only specifies a license fee $\underline{L}_{2}^{P}=\bar{\tau}_{2}^{F B}$.

\section{E.2 Tax revenue}

(i) full-separating and full commitment when the stock is exhausted:

Let us denote $\tau_{t}^{F S}$ the tax revenue collected when the parameters are such that both incentive constraints bind, both firms exhaust the stock and there is full separation:

$$
\begin{aligned}
& \underline{\tau}_{1}^{F S}+\delta \underline{\tau}_{2}^{F S}=\underline{\tau}_{1}^{F C * *}+\delta \underline{\tau}_{2}^{F C * *}+\Delta \theta(1-\delta)\left(\bar{q}_{1_{e}}^{F C}-\bar{q}_{1_{e}}^{F S}\right)-\frac{b(1+\delta)}{2}\left(\underline{q}_{1_{e}}^{F B}-\underline{q}_{1_{e}}^{F S}\right)^{2} \\
& \bar{\tau}_{1}^{F S}+\delta \bar{\tau}_{2}^{F S}=\bar{\tau}_{1}^{F C * *}+\delta \bar{\tau}_{2}^{F C * *}-\frac{b(1+\delta)}{2}\left(\bar{q}_{1_{e}}^{F C}-\bar{q}_{1_{e}}^{F S}\right)\left(2 \bar{q}_{1_{e}}^{F B}-\bar{q}_{1_{e}}^{F C}-\bar{q}_{1_{e}}^{F S}\right)
\end{aligned}
$$

As compared to the full commitment case, the tax loss is given by:

$\frac{b(1+\delta)\left(1-\nu_{1}\right) \nu_{1}}{2\left(1-(2-\delta) \delta \nu_{1}\right)}\left(\underline{q}_{1_{e}}^{F B}-\bar{q}_{1_{e}}^{F C}-\delta\left(S-\bar{q}_{1_{e}}^{F C}\right)\right)^{2}$

(ii) full pooling and full commitment when the stock is exhausted:

Let us denote $\tau_{t}^{F S}$ the tax revenue collected when the parameters are such that both incentive 
constraints bind, both firms exhaust the stock and and there is full separation:

$$
\begin{aligned}
\underline{\tau}_{1}^{P}+\delta \underline{\tau}_{2}^{P} & =\underline{\tau}_{1}^{F C * *}+\delta \underline{\tau}_{2}^{F C * *}-\Delta \theta\left(\bar{q}_{1_{e}}^{F C}+\delta\left(\bar{q}_{1_{e}}^{F B}-\bar{q}_{1_{e}}^{F C}\right)\right)-\left(u\left(\bar{\theta}, \bar{q}_{1_{e}}^{F B}, 0, p_{1}\right)-u\left(\underline{\theta}, \underline{q}_{1_{e}}^{F B}, 0, p_{1}\right)\right) \\
& +\delta\left(u\left(\underline{\theta}, S-\bar{q}_{1_{e}}^{F B}, 0, p_{2}\right)-u\left(\underline{\theta}, S-\underline{q}_{1_{e}}^{F B}, 0, p_{2}\right)\right) \\
\bar{\tau}_{1}^{P}+\delta \bar{\tau}_{2}^{P} & =\bar{\tau}_{1}^{F C * *}+\delta \bar{\tau}_{2}^{F C * *}+\frac{b(1+\delta)}{2}\left(\bar{q}_{1_{e}}^{F B}-\bar{q}_{1_{e}}^{F C}\right)^{2}
\end{aligned}
$$

By simplifying, we can easily show that as compared to the full commitment case, the tax loss is given by: $\frac{(1-\delta)^{2} \nu_{1} \Delta \theta^{2}}{2 b(1+\delta)\left(1-\nu_{1}\right)}$

(iii) full-separating and pooling contracts:

The regulator proposes a pooling contract if the cost of full separation is larger than the cost of pooling, that is if:

$$
\begin{array}{r}
\frac{b(1+\delta)\left(1-\nu_{1}\right) \nu_{1}}{2\left(1-(2-\delta) \delta \nu_{1}\right)}\left(\underline{q}_{1_{e}}^{F B}-\bar{q}_{1_{e}}^{F C}-\delta\left(S-\bar{q}_{1_{e}}^{F C}\right)\right)^{2}>\frac{(1-\delta)^{2} \nu_{1} \Delta \theta^{2}}{2 b(1+\delta)\left(1-\nu_{1}\right)} \\
\frac{b(1+\delta)\left(1-\nu_{1}\right) \nu_{1}}{2\left(1-(2-\delta) \delta \nu_{1}\right)}\left(\frac{\Delta \theta(1-\delta)^{2}+\delta\left(1-\nu_{1}\right)\left(\underline{Z}-(1+\delta) \underline{X}_{2}\right)}{b(1+\delta)\left(1-\nu_{1}\right)}\right)^{2}>\frac{(1-\delta)^{2} \nu_{1} \Delta \theta^{2}}{2 b(1+\delta)\left(1-\nu_{1}\right)}
\end{array}
$$

Using the equation above, we define $\delta_{2}$ that is such that the cost of pooling equals to the cost of full separation. By rearranging the terms, we define $\delta_{2}$ that is such that computed at $\delta=\delta_{2}$, (58) is equal to zero.

$$
\left(\Delta \theta(1-\delta)^{2}+\delta\left(1-\nu_{1}\right)\left(\underline{Z}-(1+\delta) \underline{X}_{2}\right)\right)^{2}-\Delta \theta^{2}\left(1-(2-\delta) \delta \nu_{1}\right)(1-\delta)^{2}
$$

For $\delta>\delta_{2}$, a pooling contract is implemented. Note that for $\delta=1$, the cost of pooling the firm is higher than the cost of full separation, thus $\delta_{2}<1$. When countervailing incentives start to kick in, $\underline{q}_{1_{e}}^{F B}-\bar{q}_{1_{e}}^{F C}-\delta\left(S-\bar{q}_{1_{e}}^{F C}\right)=0$, and full-separating contracts are implemented. 


\section{F No stock constraint}

\section{No countervailing incentive:}

$\frac{\partial V_{R}^{N C}}{\partial \bar{q}_{1}}=-\nu_{1} x \Delta \theta+\left(1-\nu_{1} x\right)\left(\bar{X}_{1}-b \bar{q}_{1}\right)=0$ gives $\bar{q}_{1}^{N C}=\bar{q}_{1}^{F B}-\frac{\Delta \theta \nu_{1} x}{b\left(1-\nu_{1} x\right)}$. From the envelope theorem, $\frac{\partial \bar{q}_{1}^{N C}}{\partial x}=0$. However, from $\bar{q}_{2 / \bar{C}_{1}}^{N C}=\bar{q}_{2}^{F B}-\frac{\Delta \theta \nu_{1}(1-x)}{b\left(1-\nu_{1}\right)}, \frac{\partial \bar{q}_{2 / \bar{C}_{1}}^{N C}}{\partial x}=\frac{\Delta \theta \nu_{1}}{b\left(1-\nu_{1}\right)}$.

We can easily show that there is full separation $(F S)$ since the tax revenue is higher than under semi-separation $\left(S S^{x}\right)$ and under full pooling $(F P)$. Indeed, computed at the equilibrium: $V_{R}^{F S}-V_{R}^{S S^{x}}=\frac{\Delta \theta^{2} \nu_{1}\left(\delta \nu_{1} x^{2}\left(1-\nu_{1} x\right)+1-x\right)}{2 b\left(1-\nu_{1}\right)\left(1-\nu_{1} x\right)}>0$ and $V_{R}^{F S}-V_{R}^{P}=\frac{\Delta \theta^{2} \nu_{1}}{2 b\left(1-\nu_{1}\right)}>0$

Without commitment the welfare is lower than with commitment: $V_{R}^{F C}-V_{R}^{N C}=\frac{\delta \Delta \theta^{2} \nu_{1}^{2}}{2 b\left(1-\nu_{1}\right)}>0$

\section{Countervailing incentive:}

By replacing $\underline{\tau}_{1}^{N C}=\underline{\tau}_{1}^{R}-\Delta \theta\left(\bar{q}_{1}+\delta \bar{q}_{2 / \bar{C}_{1}}-\delta \bar{q}_{2 / \underline{C}_{1}}\right), \bar{\tau}_{t}^{N C}=\bar{\tau}_{t}^{R}$, and $\underline{q}_{1}=\bar{q}_{1}+\delta\left(\bar{q}_{2 / \bar{C}_{1}}-\bar{q}_{2 / \underline{C}_{1}}\right)$ into (25), the regulator's problem becomes:

$$
\begin{aligned}
\max _{\left\{\bar{q}_{1}, x, y\right\}} V_{R}^{N C} & =\left(\nu_{1} x+\left(1-\nu_{1}\right) y\right)\left(\underline{\tau}_{1}^{R}-\Delta \theta \underline{q}_{1}\right)+\left(\nu_{1}(1-x)+\left(1-\nu_{1}\right)(1-y)\right) \bar{\tau}_{1}^{R} \\
& +\delta\left(1-\nu_{1}\right)\left((1-y) \bar{\tau}_{2 / \bar{C}_{1}}^{R}+y \bar{\tau}_{2 / \underline{C}_{1}}^{R}\right) \\
& +\delta \nu_{1}\left(x\left(\underline{\tau}_{2 / \underline{C}_{1}}^{R}-\Delta \theta \bar{q}_{2 / \underline{C}_{1}}\right)+(1-x)\left(\underline{\tau}_{2 / \bar{C}_{1}}^{R}-\Delta \theta \bar{q}_{2 / \bar{C}_{1}}\right)\right)
\end{aligned}
$$

By deriving (59) with respect to $\bar{q}_{1}$, we get:

$$
\begin{aligned}
\frac{\partial V_{R}^{N C}}{\partial \bar{q}_{1}} & =\bar{X}_{1}-b \bar{q}_{1}-\delta\left(\nu_{1} x+\left(1-\nu_{1}\right) y\right)\left(\bar{q}_{2 / \bar{C}_{1}}-\bar{q}_{2 / \underline{C}_{1}}\right)=0 \\
\bar{q}_{1}^{N C} & =\bar{q}_{1}^{F B}-\delta\left(\nu_{1} x+\left(1-\nu_{1}\right) y\right)\left(\bar{q}_{2 / \bar{C}_{1}}-\bar{q}_{2 / \underline{C}_{1}}\right) \\
\underline{q}_{1}^{N C} & =\bar{q}_{1}^{F B}+\delta\left(\nu_{1}(1-x)+\left(1-\nu_{1}\right)(1-y)\right)\left(\bar{q}_{2 / \bar{C}_{1}}-\bar{q}_{2 / \underline{C}_{1}}\right)
\end{aligned}
$$

Under full separation , $x=1$ and $y=0$, thus, $\bar{q}_{2 / \bar{C}_{1}}=\bar{q}_{2}^{F B}$ and $\bar{q}_{2 / \underline{C}_{1}}=0$.

From (60) and (61), $\bar{q}_{1}^{F S_{2}}=\bar{q}_{1}^{F B}-\delta \nu_{1} \bar{q}_{2}^{F B}=\bar{q}_{1}^{F C}-\nu_{1}\left(\bar{q}_{1}^{F C}+\delta \bar{q}_{2}^{F B}-\underline{q}_{1}^{F B}\right)$ and $\underline{q}_{1}^{F S_{2}}=\bar{q}_{1}^{F B}+\delta\left(1-\nu_{1}\right) \bar{q}_{2}^{F B}=\underline{q}_{1}^{F B}+\left(1-\nu_{1}\right)\left(\bar{q}_{1}^{F C}+\delta \bar{q}_{2}^{F B}-\underline{q}_{1}^{F B}\right)$. Since there is a countervailing incentive, $\bar{q}_{1}^{F C}+\delta \bar{q}_{2}^{F B}>\underline{q}_{1}^{F B}$, thus, $\underline{q}_{1}^{F S_{2}}>\underline{q}_{1}^{F B}=\underline{q}_{1}^{F C}$ and $\bar{q}_{1}^{F S_{2}}<\bar{q}_{1}^{F C}<\bar{q}_{1}^{F B}$. 
Under semi-separating contracts where an inefficient firm randomizes $\left(S S_{2}^{y}\right): x=1$ and $y>0$, $\bar{q}_{2 / \bar{C}_{1}}=\bar{q}_{2}^{F B}$ and $\bar{q}_{2 / \underline{C}_{1}}=\bar{q}_{2}^{F B}-\frac{\nu_{1} \Delta \theta}{b\left(1-\nu_{1}\right) y}$. From (60) and (61), $\bar{q}_{1}^{S S_{2}^{y}}=\bar{q}_{1}^{F B}-\frac{\delta\left(\nu_{1}+\left(1-\nu_{1}\right) y\right) \Delta \theta \nu_{1}}{b\left(1-\nu_{1}\right) y}$ and $\underline{q}_{1}^{S S_{2}^{y}}=\bar{q}_{1}^{F B}+\frac{\delta(1-y) \Delta \theta \nu_{1}}{b y}$. The optimal level of separation is determined by solving:

$$
\begin{aligned}
\max _{y} V_{R}^{N C} & =\left(\nu_{1}+\left(1-\nu_{1}\right) y\right)\left(\underline{\tau}_{1}^{R}-\Delta \theta \underline{q}_{1}^{S S_{2}^{y}}\right)+\left(1-\nu_{1}\right)(1-y) \bar{\tau}_{1}^{R}+\delta \nu_{1}\left(\underline{\tau}_{2 / \underline{C}_{1}}^{R}-\Delta \theta \bar{q}_{2 / \underline{C}_{1}}\right) \\
& +\delta\left(1-\nu_{1}\right)\left(y \bar{\tau}_{2 / \underline{C}_{1}}^{R}+(1-y) \bar{\tau}_{2 / \bar{C}_{1}}^{R}\right)
\end{aligned}
$$

From the envelope theorem, $\frac{\partial \bar{q}_{1}^{S S_{2}^{y}}}{\partial y}=0$, and from (28), $\frac{\partial \underline{q}_{1}^{S S_{2}^{y}}}{\partial y}=-\delta \frac{\partial \bar{q}_{2 / \underline{C}_{1}}}{\partial y}=-\frac{\delta \nu_{1} \Delta \theta}{b\left(1-\nu_{1}\right) y^{2}}$.

$$
\begin{aligned}
\frac{\partial V_{R}^{N C}}{\partial y} & =\left(1-\nu_{1}\right)\left(\underline{\tau}_{1}^{R}-\Delta \theta \underline{q}_{1}^{S S_{2}^{y}}-\bar{\tau}_{1}^{R}+\delta \bar{\tau}_{2 / \underline{C}_{1}}^{R}-\delta \bar{\tau}_{2 / \bar{C}_{1}}^{R}\right) \\
& +\left(\nu_{1}+\left(1-\nu_{1}\right) y\right)\left(\frac{\partial \underline{\tau}_{1}^{R}}{\partial \underline{q}_{1}^{S S_{2}^{y}}}-\Delta \theta\right) \frac{\partial \underline{q}_{1}^{S S_{2}^{y}}}{\partial y}+\delta \frac{\partial \bar{q}_{2 / \underline{C}_{1}}}{\partial y}\left(-\nu_{1} \Delta \theta+\left(1-\nu_{1}\right) y \frac{\partial \bar{\tau}_{2 / \underline{C}_{1}}^{R}}{\partial \bar{q}_{2 / \underline{C}_{1}}}\right)
\end{aligned}
$$

After simplification, (63) can be rewritten as:

$\frac{\delta \Delta \theta^{2} \nu_{1}^{2}}{2 b\left(1-\nu_{1}\right) y^{3}}\left(2 \delta \nu_{1}(1-y)-(1-\delta) y\right)=0 \Leftrightarrow y^{S S_{2}^{y}}=\frac{2 \delta \nu_{1}}{2 \delta \nu_{1}+1-\delta}$

$\bar{q}_{1}^{S S_{2}^{y}}=\bar{q}_{1}^{F B}-\frac{(1+\delta) \Delta \theta \nu_{1}}{2 b\left(1-\nu_{1}\right)}=\bar{q}_{1}^{F C}+\frac{(1-\delta) \Delta \theta \nu_{1}}{2 b\left(1-\nu_{1}\right)} \Leftrightarrow \bar{q}_{1}^{F C}<\bar{q}_{1}^{S S_{2}^{y}}<\bar{q}_{1}^{F B}$

$\underline{q}_{1}^{S S_{2}^{y}}=\underline{q}_{1}^{F B}-\frac{(1+\delta) \Delta \theta}{2 b} \Leftrightarrow \underline{q}_{1}^{S S_{2}^{y}}<\underline{q}_{1}^{F B}=\underline{q}_{1}^{F C}$

$\bar{q}_{2 / \underline{C}_{1}}^{N C}\left(1, y^{S S_{2}^{y}}\right)=\bar{q}_{2}^{F B}-\frac{\Delta \theta\left(2 \delta \nu_{1}+1-\delta\right)}{2 b \delta\left(1-\nu_{1}\right)}=\bar{q}_{2}^{F C}-\frac{\Delta \theta(1-\delta)}{2 b \delta\left(1-\nu_{1}\right)}<\bar{q}_{2}^{F C}<\bar{q}_{2}^{F B}$

Note that $S>\underline{q}_{1}^{S S_{2}^{y}}+\underline{q}_{2}^{F B}$ implies $S>\frac{\underline{X}_{1}}{b}+\frac{\underline{X}_{2}}{b}-\frac{(1+\delta) \Delta \theta}{2 b} \Leftrightarrow S>\frac{\bar{X}_{1}}{b}+\frac{\bar{X}_{2}}{b}+\frac{(3-\delta) \Delta \theta}{2 b}$ which contradicts $S<S^{a}$.

Since $\left.\frac{\partial V_{R}^{N C}}{\partial y}\right|_{y=1}=-\frac{\delta \Delta \theta^{2} \nu_{1}^{2}}{2 b\left(1-\nu_{1}\right)}(1-\delta)<0, \forall \delta<1$, semi-separating contracts $(x, y)=\left(1, y^{*}\right)$ strictly dominate a pooling contract $(x=y=1)$. 


\section{G Exhaustion date}

\section{G.1 Three-period model}

We consider the case in which the firm extracts a positive quantity at each period and exhausts the stock at the end of the third period. We use the superscript 3 to refer to this case.

The problem is always solved backward. Hence, at the last two periods the problem is the same as the two-period model except that $X_{1}=X_{2}, X_{2}=X_{3}$ and $S=S_{2}=S-q_{1}$.

The first best: Both types exhaust the stock during the third period if $\sum_{t=1}^{t=3} \frac{\bar{X}_{t}}{b}>S$. Adapting (10) and (11), the extraction at the last two periods is:

$q_{2_{e}}^{F B_{3}}\left(S_{2}\right)=\frac{X_{2}-\delta X_{3}+\delta b S_{2}}{b(1+\delta)}$

$q_{3_{e}}^{F B_{3}}\left(S_{2}\right)=S_{2}-q_{2_{e}}^{F B_{3}}\left(S_{2}\right)=\frac{\delta X_{3}-X_{2}+b S_{2}}{b(1+\delta)}$

Note that the stock is exhausted during the second period if $q_{3_{e}}^{F B_{3}}\left(S_{2}\right)<0$, that is if:

$\delta X_{3}-X_{2}+b S_{2}<0$

We insert (64) and (65) in the regulator's objective function and solves the problem with respect to the first-period extraction: $\max _{q_{1}} V_{R}^{F B}=\tau_{1}^{R}+\delta \tau_{2}^{R}+\delta^{2} \tau_{3}^{R}$, which gives:

$q_{1_{e}}^{F B_{3}}=\frac{(1+\delta) X_{1}-\delta^{2}\left(X_{2}+X_{3}-b S\right)}{b\left(1+\delta+\delta^{2}\right)}$

To obtain the second and the third period first best level, $q_{2_{e}}^{F B_{3}}$ and $q_{3_{e}}^{F B_{3}}$, we simply replace $S_{2}=S-q_{1_{e}}^{F B_{3}}$ into (64) and (65) where $q_{1_{e}}^{F B_{3}}$ is defined in (67).

With commitment: Both firms exhaust the stock if: $\sum_{t=1}^{t=3}\left(\frac{\bar{X}_{t}}{b}-\frac{\Delta \theta \nu_{1}}{b\left(1-\nu_{1}\right)}\right)>S$.

From, the two-period problem, $\underline{\theta}$ produces the first best in the last two periods.

By adapting (23) and (24) and by denoting $\bar{S}_{2}^{F C_{3}}=S-\bar{q}_{1}^{F C_{3}}$, the inefficient firm's extraction 
during the last two periods is:

$$
\begin{aligned}
\bar{q}_{2_{e}}^{F C_{3}} & =\frac{\bar{X}_{2}-\delta \bar{X}_{3}+\delta b \bar{S}_{2}^{F C_{3}}}{b(1+\delta)}-\frac{(1-\delta) \nu_{1} \Delta \theta}{b(1+\delta)\left(1-\nu_{1}\right)} \\
\bar{q}_{3}^{F C_{3}} & =\frac{\delta \bar{X}_{3}-\bar{X}_{2}+b \bar{S}_{2}^{F C_{3}}}{b(1+\delta)}+\frac{(1-\delta) \Delta \theta \nu_{1}}{b(1+\delta)\left(1-\nu_{1}\right)}
\end{aligned}
$$

We insert the inefficient firm's production given by (68) and (69) and the efficient firm's production given by (64) and (65) in the regulator's objective function. We differentiate and solve the problem with respect to the first-period extractions:

$\max _{\bar{q}_{1}, \underline{q}_{1}} V_{R}^{F B}=\nu_{1}\left(\underline{\tau}_{1}^{R}+\delta \underline{\tau}_{2}^{R}+\delta^{2} \underline{\tau}_{3}^{R}-\Delta \theta\left(\bar{q}_{1}+\delta \bar{q}_{2}^{F C_{3}}+\delta^{2} \bar{q}_{3}^{F C_{3}}\right)\right)+\left(1-\nu_{1}\right)\left(\bar{\tau}_{1}^{R}+\delta \bar{\tau}_{2}^{R}+\delta^{2} \bar{\tau}_{3}^{R}\right)$. By solving the problem, we find that $\underline{\theta}$ produces the first best given by (67), while $\bar{\theta}$ produces:

$\bar{q}_{1_{e}}^{F C_{3}}=\bar{q}_{1_{e}}^{F B_{3}}-\frac{(1-\delta)(1+2 \delta) \Delta \theta \nu_{1}}{b\left(\delta^{2}+1+\delta\right)\left(1-\nu_{1}\right)}$

No commitment and only $(\underline{I C})$ binds: All types of firms exhaust the stock during the third period if: $\left(1-\nu_{1}\right)\left(b S-\bar{X}_{1}-\bar{X}_{2}-\bar{X}_{3}\right)+\nu_{1} \Delta \theta<0 .^{8}$

From the two-period model, we can derive the extraction of the three-period model. In the second period, full separation is optimal, and an efficient firm produces the first best given by (64). By adapting (23), that is by replacing $\nu_{1}$ by $\nu_{2}=\left\{\underline{\nu}_{2}=1, \bar{\nu}_{2}=\frac{\nu_{1}(1-x)}{1-\nu_{1} x}\right\}$ and $S$ by $S_{2}=S-q_{1}$, an inefficient firm produces: $\bar{q}_{2_{e}}^{N C_{3}}\left(S_{2}\right)=\bar{q}_{2_{e}}^{F B_{3}}\left(S_{2}\right)-\frac{\Delta \theta \nu_{2}(1-\delta)}{b\left(1-\nu_{2}\right)(1+\delta)}$.

We drop the subscript $\bar{C}_{1}$ in $\bar{z}_{t / \bar{C}_{1}}^{N C_{3}}$ where $t=\{1,2\}$ and simply write $\bar{z}_{t}^{N C_{3}}$ since the variables $\bar{z}_{t}^{N C_{3}}$ only occur if $\bar{C}_{1}$ is chosen. Indeed, if $\underline{C}_{1}$ is chosen, only $\underline{C}_{t}$ is proposed in the following periods. In the first period, the regulator's problem is:

$$
\begin{aligned}
\max _{\left\{\underline{q}_{1}, \underline{q}_{1}, x\right\}} V_{R}^{N C} & =\nu_{1} x\left(\underline{\tau}_{1}^{R}-\Delta \theta\left(\bar{q}_{1}+\delta \bar{q}_{2}^{N C_{3}}+\delta^{2} \bar{q}_{3}^{N C_{3}}\right)+\delta \underline{\tau}_{2 / \underline{C}_{1}}^{R}+\delta^{2} \underline{\tau}_{3 / \underline{C}_{1}}^{R}\right)+\left(1-\nu_{1} x\right) \bar{\tau}_{1}^{R} \\
& +\delta\left(1-\nu_{1}\right)\left(\bar{\tau}_{2}^{R}+\delta \bar{\tau}_{3}^{R}\right)+\delta \nu_{1}(1-x)\left(\underline{\tau}_{2 / \bar{C}_{1}}^{R}-\Delta \theta\left(\bar{q}_{2}^{N C_{3}}+\delta \bar{q}_{3}^{N C_{3}}\right)+\delta \underline{\tau}_{3 / \bar{C}_{1}}^{R}\right)
\end{aligned}
$$

\footnotetext{
${ }^{8}$ Without commitment, if the stock is not exhausted, during the first period, full separation is optimal, and an inefficient firm produces the same as with commitment: $\frac{\bar{X}_{1}}{b}-\frac{\Delta \theta \nu_{1}}{b\left(1-\nu_{1}\right)}$, and then produces the first best: $\left(\frac{\bar{X}_{2}}{b}, \frac{\bar{X}_{3}}{b}\right)$. Thus, both types exhaust the stock if: $\frac{\bar{X}_{1}}{b}-\frac{\Delta \theta \nu_{1}}{b\left(1-\nu_{1}\right)}+\frac{\bar{X}_{2}}{b}+\frac{\bar{X}_{3}}{b}>S$.
} 
$\bar{q}_{1_{e}}^{N C_{3}}=\bar{q}_{1_{e}}^{F B_{3}}-\frac{(1-\delta)(1+2 \delta) \Delta \theta \nu_{1} x}{b\left(\delta^{2}+1+\delta\right)\left(1-\nu_{1} x\right)}$

The optimal separation level is given by:

$\begin{aligned} \frac{\partial V_{R}^{N C}}{\partial x} & =\delta\left(1-\nu_{1}\right)\left(\frac{\partial \bar{\tau}_{2}^{R}}{\partial x}+\delta \frac{\partial \bar{\tau}_{3}^{R}}{\partial x}\right)-\delta \Delta \theta \nu_{1}\left(\frac{\partial \bar{q}_{2}^{N C_{3}}}{\partial x}+\delta \frac{\partial \bar{q}_{3}^{N C_{3}}}{\partial x}\right) \\ & +\nu_{1}\left(\underline{\tau}_{1}^{R}-\bar{\tau}_{1}^{R}-\Delta \theta \bar{q}_{1}^{N C_{3}}+\delta\left(\underline{\tau}_{2 / \underline{C}_{1}}^{R}-\underline{\tau}_{2 / \bar{C}_{1}}^{R}+\delta\left(\underline{\tau}_{3 / \underline{C}_{1}}^{R}-\underline{\tau}_{3 / \bar{C}_{1}}^{R}\right)\right)\right)\end{aligned}$

using $\frac{\partial \bar{q}_{1}^{N C_{3}}}{\partial x}=0, \frac{\partial \bar{q}_{2}^{N C_{3}}}{\partial x}=\frac{(1-\delta) \Delta \theta \nu_{1}}{b(1+\delta)\left(1-\nu_{1}\right)}$ and $\frac{\partial \bar{q}_{3}^{N C_{3}}}{\partial x}=-\frac{(1-\delta) \Delta \theta \nu_{1}}{b(1+\delta)\left(1-\nu_{1}\right)}$

$\frac{\partial V_{R}^{N C}}{\partial x}=\frac{(1-\delta)^{2} \Delta \theta^{2} \nu_{1}}{b(1+\delta)}\left(\frac{(1+2 \delta)^{2}}{2\left(\delta^{2}+1+\delta\right)\left(1-\nu_{1} x\right)^{2}}-\frac{\delta \nu_{1} x}{1-\nu_{1}}\right)>0, \forall \delta \in[0,1], x \in[0,1], \nu_{1} \in[0,1]$.

$x^{*}=1, \bar{q}_{1_{e}}^{N C_{3}}=\bar{q}_{1_{e}}^{F C_{3}}$ defined by $(70)$ and $\bar{S}_{2}^{N C}=\bar{S}_{2}^{F C}=S-\bar{q}_{1_{e}}^{F C}>\bar{S}_{2}^{F B}=S-\bar{q}_{1_{e}}^{F B}$.

An inefficient firm only reveals its type if:

$\underline{q}_{1_{e}}^{F B_{3}} \geq \bar{q}_{1_{e}}^{F C_{3}}+\delta \bar{q}_{2_{e}}^{F C_{3}}+\delta^{2}\left(S-\bar{q}_{1_{e}}^{F C_{3}}-\bar{q}_{2_{e}}^{F C_{3}}\right)$

\section{G.2 No commitment and full separation}

In what follows, we consider different cases to show that under asymmetric information without commitment, if the discount factor is relatively large, then there is a countervailing incentive and in the first period, full-separating contracts are implemented. If so, an efficient firm may exhaust the resource before the first best. On the opposite, an inefficient firm may exhaust the resource after the first best. We do not define the entire set of conditions under which these cases occur.

The inability to commit may advance the exhaustion of the resource by an efficient firm $\left(\underline{T}^{N C}<\underline{T}^{F B}\right)$.

Assume that under symmetric information, both firms exhaust the stock during the third period. Then, from (66), $\delta \underline{X}_{3}-\underline{X}_{2}+b \underline{S}_{2}^{F B}>0$ and $\delta \bar{X}_{3}-\bar{X}_{2}+b \bar{S}_{2}^{F B}>0$. Assume that without commitment, both firms also exhaust the stock during the third period, and that during the 
first period, it is optimal to fully separate firms and that both $(I C)$ bind. This implies that full-separating contracts where only $(\underline{I C})$ binds are not incentive compatible, therefore: $\underline{q}_{1_{e}}^{F B_{3}}<\bar{q}_{1_{e}}^{F C_{3}}+\delta \bar{q}_{2_{e}}^{F B_{3}}\left(\bar{S}_{2}^{F C}\right)+\delta^{2} \bar{q}_{3_{e}}^{F B_{3}}\left(\bar{S}_{2}^{F C}\right)$, where $\bar{S}_{2}^{F C}=S-\bar{q}_{1_{e}}^{F C}$, and $\underline{q}_{1_{e}}^{F B_{3}}, \bar{q}_{2_{e}}^{F B_{3}}\left(\bar{S}_{2}^{F C}\right)$, $\bar{q}_{3_{e}}^{F B_{3}}\left(\bar{S}_{2}^{F C}\right)$ and $\bar{q}_{1_{e}}^{F C_{3}}$ are respectively given by (67), (64), (65) and (70).

Under full-separating contracts where both $(I C)$ bind, if both firms exhaust the stock during the third period, it is easy to show that the efficient firm's extraction is:

$\underline{q}_{1_{e}}^{N C_{3}}=\underline{q}_{1_{e}}^{F B_{3}}+\frac{(1-\delta)\left(1-\nu_{1}\right)}{4 \delta^{2}\left(\delta^{2}-\delta-1\right) \nu_{1}+(1+\delta)^{2}}\left(\bar{q}_{1_{e}}^{F C_{3}}+\delta \bar{q}_{2_{e}}^{F B_{3}}\left(\bar{S}_{2}^{F C}\right)+\delta^{2} \bar{q}_{3_{e}}^{F B_{3}}\left(\bar{S}_{2}^{F C}\right)-\underline{q}_{1_{e}}^{F B_{3}}\right)$ $4 \delta^{2}\left(\delta^{2}-\delta-1\right) \nu_{1}+(1+\delta)^{2}>0$, and from both $(I C)$ bind $\bar{q}_{1_{e}}^{F C_{3}}+\delta \bar{q}_{2_{e}}^{F B_{3}}\left(\bar{S}_{2}^{F C}\right)+\delta^{2} \bar{q}_{3_{e}}^{F B_{3}}\left(\bar{S}_{2}^{F C}\right)-$ $\underline{q}_{1_{e}}^{F B_{3}}>0$. Hence, $\underline{q}_{1_{e}}^{N C_{3}}>\underline{q}_{1_{e}}^{F B_{3}} \Leftrightarrow \underline{S}_{2}^{N C}=S-\underline{q}_{1_{e}}^{N C}<\underline{S}_{2}^{F B}=S-\underline{q}_{1_{e}}^{F B}$ and $\delta \underline{X}_{3}-\underline{X}_{2}+b \underline{S}_{2}^{N C}<0$ is compatible with $\delta \underline{X}_{3}-\underline{X}_{2}+b \underline{S}_{2}^{F B}>0$. Under asymmetric information without commitment, it may be optimal for an efficient firm to exhaust the stock during the second period, while under symmetric information, the firm exhausts the stock during the third period.

The inability to commit may postpone the exhaustion of the resource by an inefficient firm as compared the full commitment case $\left(\bar{T}^{N C}>\bar{T}^{F C}\right)$.

Assume that with commitment, both firms exhaust the stock during the second period. Then, from (66), $\delta \underline{X}_{3}-\underline{X}_{2}+b \underline{S}_{2}^{F B}<0$, and from (69), $\left(1-\nu_{1}\right)\left(\delta \bar{X}_{3}-\bar{X}_{2}+b \bar{S}_{2}^{F C}\right)+(1-$

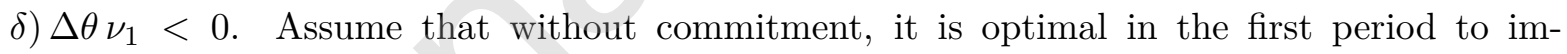
plement full-separating contracts where both $(I C)$ bind. In the second period, the firstbest contracts are implemented, and from (66), an inefficient firm exhausts the stock during the second period, if $\delta \bar{X}_{3}-\bar{X}_{2}+b \bar{S}_{2}^{N C}<0$, where $\bar{S}_{2}^{N C}=S-\bar{q}_{1_{e}}^{N C}$ and $\bar{q}_{1_{e}}^{N C}$ is given by $\bar{q}_{1_{e}}^{F S}=\bar{q}_{1_{e}}^{F C}-\frac{(1-\delta) \nu_{1}}{1-(2-\delta) \delta \nu_{1}}\left(\bar{q}_{1_{e}}^{F C}+\delta\left(S-\bar{q}_{1_{e}}^{F C}\right)-\underline{q}_{1_{e}}^{F B}\right)$. Since $\bar{q}_{1_{e}}^{N C}<\bar{q}_{1_{e}}^{F C}$ where $\bar{q}_{1_{e}}^{F C}$ is given by (23), $\bar{S}_{2}^{N C}>\bar{S}_{2}^{F C}$, and thus $\delta \bar{X}_{3}-\bar{X}_{2}+b \bar{S}_{2}^{N C}>0$ is compatible with $\left(1-\nu_{1}\right)\left(\delta \bar{X}_{3}-\bar{X}_{2}+b \bar{S}_{2}^{F C}\right)+(1-\delta) \Delta \theta \nu_{1}<0$. In other word, $\bar{q}_{3_{e}}^{F B}\left(\bar{S}_{2}^{N C}\right)>0$ is compatible with $\bar{q}_{3_{e}}^{F C}\left(\bar{S}_{2}^{F C}\right)<0$, and thus, without commitment, an inefficient firm may exhaust the stock during the third period, while with commitment, it exhausts the stock during the second period. 
Note that under full-separating contracts where both $(I C)$ bind, if an efficient firm exhausts the stock during the second period, while an inefficient firm exhausts the stock during the third period, it is easy to show that the first-period extractions are:

$\underline{q}_{1_{e}}^{N C}=\underline{q}_{1_{e}}^{F B}+\frac{\left(\delta^{2}+1+\delta\right)\left(1-\nu_{1}\right)}{\left(\delta-4 \delta^{2}\left(1-\delta^{2}+\delta\right)\right) \nu_{1}+(1+\delta)^{2}}\left(\bar{q}_{1_{e}}^{F C_{3}}+\delta \bar{q}_{2_{e}}^{F B_{3}}\left(\bar{S}_{2_{e}}^{F C}\right)+\delta^{2} \bar{q}_{3_{e}}^{F B_{3}}\left(\bar{S}_{2}^{F C}\right)-\underline{q}_{1_{e}}^{F B}\right)$

$\bar{q}_{1_{e}}^{N C_{3}}=\bar{q}_{1_{e}}^{F C_{3}}-\frac{(1-\delta)(1+\delta)(1+2 \delta) \nu_{1}}{\left(\delta-4 \delta^{2}\left(1-\delta^{2}+\delta\right)\right) \nu_{1}+(1+\delta)^{2}}\left(\bar{q}_{1_{e}}^{F C_{3}}+\delta \bar{q}_{2_{e}}^{F B_{3}}\left(\bar{S}_{2}^{F C}\right)+\delta^{2} \bar{q}_{3_{e}}^{F B_{3}}\left(\bar{S}_{2}^{F C}\right)-\underline{q}_{1_{e}}^{F B}\right)$

\section{G.3 No commitment and full pooling}

In what follows, we consider different cases to show that under asymmetric information without commitment, if the discount factor is large, then a countervailing incentive exists, and in the first period, a pooling contract is implemented. An efficient firm may exhaust the stock at a later period, while the inefficient firm's extraction is the same as under symmetric information. We do not define the entire set of conditions under which these cases occur.

The inability to commit may postpone the exhaustion of the resource by an efficient firm $\left(\underline{T}^{N C}>\underline{T}^{F B}\right)$.

Assume that under symmetric information, it is optimal for an efficient firm to exhaust the stock during the second period, and for an inefficient firm to exhaust the stock during the third period. Then, from (66), $\delta \underline{X}_{3}-\underline{X}_{2}+b \underline{S}_{2}^{F B}<0$ and $\delta \bar{X}_{3}-\bar{X}_{2}+b \bar{S}_{2}^{F B}>0$. If without commitment, it is optimal to propose during the first period a pooling contract, and during the second period full-separating contracts where only $(\underline{I C})$ binds, then, it may be optimal for an efficient firm to exhaust the stock during the third period. If so, the first-period extraction is $\bar{q}_{1_{e}}^{F B}$ given by (67), and $\bar{q}_{1_{e}}^{F B}<\underline{q}_{1_{e}}^{F B}$ implies $\bar{S}_{2}^{F B}>\underline{S}_{2}^{F B}$. Thus, $\delta \underline{X}_{3}-\underline{X}_{2}+b \bar{S}_{2}^{F B}>0$ is compatible with $\delta \underline{X}_{3}-\underline{X}_{2}+b \underline{S}_{2}^{F B}<0$.

Note that if during the first period a pooling contract is implemented, and during the second period, full-separating contracts where only $(\underline{I C})$ binds are proposed, if an efficient firm exhausts the stock during the second period, while an inefficient firm exhausts the stock during the third period, it is easy to show that the first-period extraction $q_{1_{e}}^{p}$ is given by the following first-order condition: $\frac{(1+\delta) \bar{X}_{1}-\delta^{2}\left(\bar{X}_{3}+\bar{X}_{2}-b S\right)-\left(\delta^{2}+1+\delta\right) q_{1 e}^{p}}{1+\delta}+\frac{\delta \nu_{1}\left(\delta \underline{X}_{3}-\underline{X}_{2}+b\left(S-q_{1}^{p}\right)\right)}{1+\delta}=0$, which implies from $\delta \underline{X}_{3}-\underline{X}_{2}+b\left(S-q_{1_{e}}^{p}\right)<0$ and $(67)$, that $q_{1_{e}}^{P}<\bar{q}_{1_{e}}^{F B}$. 
The inability to commit may advance the exhaustion of the resource by an inefficient firm as compared the full commitment case $\left(\bar{T}^{N C}<\bar{T}^{F C}\right)$.

Assume that under information asymmetry with commitment, an efficient firm exhausts the stock during the second period, while an inefficient firm exhausts the stock during the third period. Then, from (66), $\delta \underline{X}_{3}-\underline{X}_{2}+b \underline{S}_{2}^{F B}<0$, and from (69), $\left(1-\nu_{1}\right)\left(\delta \bar{X}_{3}-\bar{X}_{2}+b \bar{S}_{2}^{F C}\right)+$ $(1-\delta) \Delta \theta \nu_{1}>0$. Assume that without commitment, during the first period, it is optimal to propose full-separating contracts where only $(\underline{I C})$ binds. If the inefficient firm exhausts the stock during the third period, in the first period, it produces the same as with commitment, that is $\bar{q}_{1_{e}}^{F C}$ given by (70). However, in the second period, it produces the first best: $\bar{q}_{2_{e}}^{F B}\left(S_{2}^{F C}\right)$ given by (64), and thus, more than with commitment. Hence, the stock available at the end of the second period is lower without commitment. Since $\delta \bar{X}_{3}-\bar{X}_{2}+b \bar{S}_{2}^{F C}<0$ is compatible with $(1-\delta) \Delta \theta \nu_{1}+\left(1-\nu_{1}\right)\left(\delta \bar{X}_{3}-\bar{X}_{2}+b \bar{S}_{2}^{F C}\right)>0$, it may be optimal for an inefficient firm to exhaust the stock during the second period without commitment, while it exhausts the stock during the third period with commitment. 